\title{
BINARY QUARTIC FORMS WITH BOUNDED INVARIANTS AND SMALL GALOIS GROUPS
}

\author{
CINDY (SIN YI) TSANG AND STANLEY YAO XIAO
}

\begin{abstract}
In this paper, we consider integral and irreducible binary quartic forms whose Galois group is isomorphic to a subgroup of the dihedral group of order eight. We first show that the set of all such forms is a union of families indexed by integral binary quadratic forms $f(x, y)$ of non-zero discriminant. Then, we shall enumerate the $\mathrm{GL}_{2}(\mathbb{Z})$-equivalence classes of all such forms associated to a fixed $f(x, y)$.
\end{abstract}

\section{Contents}

1. Introduction 1

2. Characterization of forms with small Galois groups 8

3. Basic properties of forms in $V_{\mathbb{R}, f}$ of non-zero discriminant 10

4. Parametrizing forms in $V_{\mathbb{R}, f}$ of non-zero discriminant 15

5. Definition of a bounded semi-algebraic set 20

6. Error estimates and the main theorem 27

7. Acknowledgments 35

References $\quad 35$

\section{INTRODUCTION}

The problem of enumerating $\mathrm{GL}_{2}(\mathbb{Z})$-equivalence classes of integral and irreducible binary forms of a fixed degree has a long history. The quadratic and cubic cases were solved in $[16,22]$ and $[12,13]$, respectively, where the forms are ordered by the natural height, namely the discriminant $\Delta(-)$. The quartic case turns out to be much more challenging because while the ring of polynomial invariants for both binary quadratic and cubic forms is generated by $\Delta(-)$ as an algebra, that for binary quartic forms is generated by two independent invariants, usually denoted by $I(-)$ and $J(-)$. For

$$
F(x, y)=a_{4} x^{4}+a_{3} x^{3} y+a_{2} x^{2} y^{2}+a_{1} x y^{3}+a_{0} y^{4},
$$

they are given by the explicit formulae

$$
\begin{aligned}
& I(F)=12 a_{4} a_{0}-3 a_{3} a_{1}+a_{2}^{2}, \\
& J(F)=72 a_{4} a_{2} a_{0}+9 a_{3} a_{2} a_{1}-27 a_{4} a_{1}^{2}-27 a_{3}^{2} a_{0}-2 a_{2}^{3},
\end{aligned}
$$

which are of degrees two and three, respectively. In [4], instead of using the discriminant, Bhargava and Shankar introduced the height function

$$
H_{\mathrm{BS}}(F)=\max \left\{|I(F)|^{3}, J(F)^{2} / 4\right\} .
$$

For $X>0$, let us define

$$
\begin{aligned}
& N_{\mathbb{Z}}(X)=\#\{[F]: \text { integral and irreducible binary } \\
& \text { quartic forms } \left.F \text { such that } H_{\mathrm{BS}}(F) \leq X\right\},
\end{aligned}
$$

Date: November 13, 2018. 
where $[-]$ denotes $\mathrm{GL}_{2}(\mathbb{Z})$-equivalence class. In [4], they proved that

$$
N_{\mathbb{Z}}(X)=\frac{44 \zeta(2)}{135} X^{5 / 6}+O_{\epsilon}\left(X^{3 / 4+\epsilon}\right) \text { for any } \epsilon>0
$$

This is the first result ever obtained, and as far as we know, the only known result in the literature, for the quartic case.

1.1. Set-up and notation. In this paper, we shall also be interested in the quartic case, but only the integral and irreducible binary quartic forms $F$ with small Galois group $\operatorname{Gal}(F)$, which is defined to be the Galois group of the splitting field of $F(x, 1)$ over $\mathbb{Q}$. We know that $\operatorname{Gal}(F)$ is isomorphic to one of the following:

$$
\begin{aligned}
& S_{4}=\text { the symmetric group on four letters, } \\
& A_{4}=\text { the alternating group on four letters, } \\
& D_{4}=\text { the dihedral group of order eight, } \\
& C_{4}=\text { the cyclic group of order four, } \\
& V_{4}=\text { the Klein-four group. }
\end{aligned}
$$

We shall say that $\operatorname{Gal}(F)$ is small if it is isomorphic to $D_{4}, C_{4}$, or $V_{4}$. Recall that the cubic resolvent of $F$ is defined by

$$
\mathcal{Q}_{F}(x)=x^{3}-3 I(F) x+J(F) .
$$

Then, equivalently, we have the classical characterization that for irreducible $F$

$\operatorname{Gal}(F)$ is small if and only if $\mathcal{Q}_{F}(x)$ is reducible.

It turns out that whether $\operatorname{Gal}(F)$ is small or not may also be characterized in terms of binary quadratic forms and the following so-called twisted action of $\mathrm{GL}_{2}(\mathbb{R})$.

Given a complex binary form $\xi(x, y)$, let $\mathrm{GL}_{2}(\mathbb{R})$ act on it via

$$
\xi_{T}(x, y)=\frac{1}{\operatorname{det}(T)^{\operatorname{deg} \xi / 2}} \xi\left(t_{1} x+t_{2} y, t_{3} x+t_{4} y\right) \text { for } T=\left(\begin{array}{cc}
t_{1} & t_{2} \\
t_{3} & t_{4}
\end{array}\right) .
$$

Observe that this is only an action up to $\operatorname{sign}$ when $\operatorname{deg} \xi$ is odd, in the sense that for $T_{1}, T_{2} \in \mathrm{GL}_{2}(\mathbb{R})$, we only have $\xi_{T_{1} T_{2}}= \pm\left(\xi_{T_{1}}\right)_{T_{2}}$ in general. Now, given a real binary quadratic form $f(x, y)=\alpha x^{2}+\beta x y+\gamma y^{2}$ with $\Delta(f) \neq 0$, write

$$
M_{f}=\left(\begin{array}{cc}
\beta & 2 \gamma \\
-2 \alpha & -\beta
\end{array}\right)
$$

for its associated matrix in $\mathrm{GL}_{2}(\mathbb{R})$. Its action on binary quartic forms clearly remain unchanged if we scale $f(x, y)$ by a constant in $\mathbb{R}^{\times}$. In [27], the second-named author proved that for any real binary quartic form $F$ with $\Delta(F) \neq 0$, elements of

$$
\left\{T \in \mathrm{GL}_{2}(\mathbb{R}): T \text { is not a scalar multiple of } I_{2 \times 2} \text { and } F_{T}=F\right\}
$$

all arise from binary quadratic forms in this way; see Proposition 2.1. Recall that an integral binary quadratic form is called primitive if its coefficients are coprime. Using this result from [27], in Section 2, we shall first show that:

Theorem 1.1. Let $F$ be an integral binary quartic form with $\Delta(F) \neq 0$. Then, the following are equivalent.

(1) $\mathcal{Q}_{F}(x)$ is reducible. 
(2) $F_{T}=F$ for some $T \in \mathrm{GL}_{2}(\mathbb{Q})$ which is not a scalar multiple of $I_{2 \times 2}$.

(3) $F_{M_{f}}=F$ for an integral and primitive binary quadratic form $f$ with $\Delta(f) \neq 0$.

Moreover, in the case that $\mathcal{Q}_{F}(x)$ is reducible:

(a) If $\Delta(F) \neq \square$, then there is a unique such $f$ up to sign.

(b) If $\Delta(F)=\square$, then there are exactly three such $f$ up to sign, among which one is definite and two are indefinite.

Given a real binary quadratic form $f(x, y)$ with $\Delta(f) \neq 0$, let us further make the following definitions. First put

$$
\begin{aligned}
& V_{\mathbb{R}, f}=\left\{\text { real binary quartic forms } F \text { such that } F_{M_{f}}=F\right\}, \\
& V_{\mathbb{Z}, f}=\left\{\text { integral binary quartic forms } F \text { such that } F_{M_{f}}=F\right\} .
\end{aligned}
$$

Clearly $V_{\mathbb{R}, f}$ is a vector space over $\mathbb{R}$ and $V_{\mathbb{Z}, f}$ a lattice over $\mathbb{Z}$. A straightforward calculation shows that $\operatorname{dim}_{\mathbb{R}} V_{\mathbb{R}, f}$ is three; see (3.1) and (3.2) below. Also, put

$$
V_{\mathbb{R}, f}^{0}=\left\{F \in V_{\mathbb{R}, f}: \Delta(F) \neq 0\right\} \text { and } V_{\mathbb{Z}, f}^{0}=\left\{F \in V_{\mathbb{Z}, f}: \Delta(F) \neq 0\right\} .
$$

For $F \in V_{\mathbb{R}, f}^{0}$, we shall define two new invariants as follows. As we shall see in (2.3), there is a unique root $\omega_{f}(F)$ of $\mathcal{Q}_{F}(x)$ corresponding to $f$. Let $\omega_{f}^{\prime}(F), \omega_{f}^{\prime \prime}(F)$ denote the other two roots of $\mathcal{Q}_{F}(x)$ and define

$$
L_{f}(F)=\omega_{f}(F) \text { and } K_{f}(F)=-\omega_{f}^{\prime}(F) \omega_{f}^{\prime \prime}(F) .
$$

By Proposition 3.2 below, they have degrees one and two, respectively, in the coefficients of $F$. Following (1.2), let us define the height of $F$ associated to $f$ by

$$
H_{f}(F)=\max \left\{L_{f}(F)^{2},\left|K_{f}(F)\right|\right\} .
$$

This is comparable to the height (1.2) because by comparing coefficients in

$$
x^{3}-I(F) x+J(F)=\left(x-\omega_{f}(F)\right)\left(x-\omega_{f}^{\prime}(F)\right)\left(x-\omega_{f}^{\prime \prime}(F)\right),
$$

we easily deduce the relations

$$
3 I(F)=L_{f}(F)^{2}+K_{f}(F) \text { and } J(F)=L_{f}(F) K_{f}(F),
$$

which in turn imply that

$$
\left(H_{f}(F) / 10\right)^{3} \leq H_{\mathrm{BS}}(F) \leq H_{f}(F)^{3} .
$$

Let us note that

$$
\Delta(F)=\frac{4 I(F)^{3}-J(F)^{2}}{27}=\left(\frac{L_{f}(F)^{2}+4 K_{f}(F)}{9}\right)\left(\frac{2 L_{f}(F)^{2}-K_{f}(F)}{9}\right)^{2}
$$

where the first equality is well-known, and the second equality holds by (1.5). Also, our height $H_{f}(-)$ is an invariant in the sense that for any $T \in \mathrm{GL}_{2}(\mathbb{R})$, we have

$$
H_{f_{T}}\left(F_{T}\right)=H_{f}(F),
$$

as shown in Proposition 3.1 below. This implies that the map

$$
V_{\mathbb{R}, f} \longrightarrow V_{\mathbb{R}, f_{T}} ; \quad F \mapsto F_{T},
$$

which is a well-defined bijection because $M_{f_{T}}=T^{-1} M_{f} T$, is height-preserving when restricted to the forms of non-zero discriminant. 
Now, let us return to the integral and irreducible binary quartic forms with small Galois group. Write $V_{\mathbb{Z}}^{\text {sm }}$ for the set of all such forms and set

$$
V_{\mathbb{Z}}^{\mathrm{sm}, \dagger}=\left\{F \in V_{\mathbb{Z}}^{\mathrm{sm}}: \operatorname{Gal}(F) \neq V_{4}\right\} .
$$

By Theorem 1.1, we know that

$$
\begin{aligned}
V_{\mathbb{Z}}^{\mathrm{sm}} & =\bigcup_{f \in \mathfrak{F}^{*}}\left\{F \in V_{\mathbb{Z}, f}^{0}: F \text { is irreducible }\right\}, \\
V_{\mathbb{Z}}^{\mathrm{sm}, \dagger} & =\bigsqcup_{f \in \mathfrak{F}^{*}}\left\{F \in V_{\mathbb{Z}, f}^{0}: F \text { is irreducible and } \operatorname{Gal}(F) \neq V_{4}\right\},
\end{aligned}
$$

where $\mathfrak{F}^{*}$ denotes the set of all integral and primitive binary quadratic forms of nonzero discriminant, up to sign. In particular, given $F \in V_{\mathbb{Z}}^{\mathrm{sm}, \dagger}$, there is a unique $f \in \mathfrak{F}^{*}$ such that $F \in V_{\mathbb{Z}, f}^{0}$, and we may define the height of $F$ by setting

$$
H(F)=H_{f}(F) .
$$

For $X>0$, let us define

$$
\begin{aligned}
N_{\mathbb{Z}}^{\dagger}(X) & =\#\left\{[F]: F \in V_{\mathbb{Z}}^{\text {sm, }} \text { such that } H(F) \leq X\right\}, \\
N_{\mathbb{Z}, f}^{\dagger}(X) & =\#\left\{[F]: F \in V_{\mathbb{Z}}^{\text {sm, }} \cap V_{\mathbb{Z}, f}^{0} \text { such that } H(F) \leq X\right\} .
\end{aligned}
$$

Then, by (1.8) and (1.9), we have

$$
N_{\mathbb{Z}}^{\dagger}(X)=\sum_{f \in \mathfrak{F}} N_{\mathbb{Z}, f}^{\dagger}(X)
$$

where $\mathfrak{F}$ denotes a set of representatives of the $\mathrm{GL}_{2}(\mathbb{Z})$-equivalence classes on $\mathfrak{F}^{*}$. In Theorem 1.2, which is our main result, for $f \in \mathfrak{F}^{*}$, we shall determine the asymptotic formula for $N_{\mathbb{Z}, f}^{\dagger}(X)$. In fact, we shall consider the finer counts

$$
\begin{aligned}
& N_{\mathbb{Z}, f}^{\left(D_{4}\right)}(X)=\#\left\{[F]: F \in V_{\mathbb{Z}}^{\text {sm }} \cap V_{\mathbb{Z}, f}^{0} \text { such that } \operatorname{Gal}(F) \simeq D_{4} \text { and } H(F) \leq X\right\}, \\
& N_{\mathbb{Z}, f}^{\left(C_{4}\right)}(X)=\#\left\{[F]: F \in V_{\mathbb{Z}}^{\text {sm }} \cap V_{\mathbb{Z}, f}^{0} \text { such that } \operatorname{Gal}(F) \simeq C_{4} \text { and } H(F) \leq X\right\}, \\
& N_{\mathbb{Z}, f}^{\left(V_{4}\right)}(X)=\#\left\{[F]: F \in V_{\mathbb{Z}}^{\text {sm }} \cap V_{\mathbb{Z}, f}^{0} \text { such that } \operatorname{Gal}(F) \simeq V_{4} \text { and } H_{f}(F) \leq X\right\},
\end{aligned}
$$

and show that the latter two are negligible compared to $N_{\mathbb{Z}, f}^{\left(D_{4}\right)}(X)$. This means that most of the forms in $V_{\mathbb{Z}}^{\text {sm }} \cap V_{\mathbb{Z}, f}^{0}$ have Galois group isomorphic to $D_{4}$. However, all of our error estimates depend upon $f$. Currently, we do not know how to control them in a uniform way, and so we are unable to obtain an asymptotic formula for $N_{\mathbb{Z}}^{\dagger}(X)$ by summing over $f \in \mathfrak{F}$.

Finally, let us explain, for each $f \in \mathfrak{F}^{*}$, how counting forms in $V_{\mathbb{Z}}^{\text {sm }} \cap V_{\mathbb{Z}, f}^{0}$ may be reduced to counting lattice points. Write $f(x, y)=\alpha x^{2}+\beta x y+\gamma y^{2}$ with $\alpha, \beta, \gamma \in \mathbb{Z}$. By (3.1) and (3.2), the set $V_{\mathbb{R}, f}$ is a vector space isomorphic to $\mathbb{R}^{3}$ via

$$
\begin{array}{ll}
\Theta_{1}: a_{4} x^{4}+a_{3} x^{3} y+a_{2} x^{2} y^{2}+a_{1} x y^{3}+a_{0} y^{4} \mapsto\left(a_{4}, a_{3}, a_{2}\right) & \text { if } \alpha \neq 0, \\
\Theta_{2}: a_{4} x^{4}+a_{3} x^{3} y+a_{2} x^{2} y^{2}+a_{1} x y^{3}+a_{0} y^{4} \mapsto\left(a_{4}, a_{2}, a_{0}\right) & \text { if } \beta, \beta^{2}+4 \alpha \gamma \neq 0 .
\end{array}
$$

Recall that the subset $V_{\mathbb{Z}, f}$ has the structure of a rank-three $\mathbb{Z}$-lattice, which may be identified with the lattices

$$
\Lambda_{f, 1}=\Theta_{1}\left(V_{\mathbb{Z}, f}\right) \text { and } \Lambda_{f, 2}=\Theta_{2}\left(V_{\mathbb{Z}, f}\right)
$$


in $\mathbb{Z}^{3}$. Let us mention here that we shall use the isomorphism

$$
\Theta_{w(f)}, \text { where } w(f)= \begin{cases}1 & \text { if } f \text { is irreducible, } \\ 2 & \text { if } f \text { is reducible. }\end{cases}
$$

Thus, the problem is reduced to counting points in $\Lambda_{f, 1}$ or $\Lambda_{f, 2}$, and then sieving out those which come from reducible forms. In turn, counting lattice points amounts to computing certain volumes by a result of Davenport [11]; see Proposition 5.1.

1.2. Statement of the main theorem. It is clear that we may choose the set $\mathfrak{F}$ of representatives to be such that for all $f \in \mathfrak{F}$, the $x^{2}$-coefficient is positive, and

$$
f(x, y)=\alpha x^{2}+\beta x y, \text { where } \operatorname{gcd}(\alpha, \beta)=1 \text { and } 0<\alpha \leq \beta
$$

when $f$ is reducible. Let $\sim$ denote $\mathrm{GL}_{2}(\mathbb{Z})$-equivalence. Then, our main result is:

Theorem 1.2. Let $f(x, y)$ be an integral and primitive binary quadratic form of nonzero discriminant and with positive $x^{2}$-coefficient. Write $D_{f}=|\Delta(f)|$, and put

$$
s_{f}= \begin{cases}8 & \text { if } D_{f} \text { is odd } \\ 1 & \text { if } D_{f} \text { is even }\end{cases}
$$

(a) Suppose that $f$ is positive definite. Then, we have

$$
N_{\mathbb{Z}, f}^{\left(D_{4}\right)}(X)=\frac{1}{s_{f} r_{f}} \frac{13 \pi}{27 D_{f}^{3 / 2}} X^{3 / 2}+O_{f}\left(X^{1+\epsilon}\right) \text { for any } \epsilon>0
$$

where

$$
r_{f}=\left\{\begin{array}{cc}
6 & \text { if } f(x, y) \sim x^{2}+x y+y^{2} \\
2 & \text { if } f(x, y) \sim a x^{2}+c y^{2} \\
\quad \text { or } f(x, y) \sim a x^{2}+b x y+a y^{2} \text { with } a \neq b \\
1 \quad \text { otherwise. }
\end{array}\right.
$$

(b) Suppose that $f$ is reducible and that $f$ has the shape (1.11). Then, we have

$$
N_{\mathbb{Z}, f}^{\left(D_{4}\right)}(X)=\frac{1}{s_{f} r_{f}} \frac{8}{9 \beta^{3 / 2}} X^{3 / 2} \log X+O_{f}\left(X^{3 / 2}\right),
$$

where

$$
r_{f}= \begin{cases}1 & \text { if } \beta \nmid \alpha^{2}+1 \text { and } \beta \nmid \alpha^{2}-1, \\ 2 & \text { otherwise. }\end{cases}
$$

(c) Suppose that $f$ is indefinite and irreducible. Define $t_{D_{f}} \in \mathbb{R}$ to be such that $e^{t_{D_{f}}}$ is the fundamental unit of the quadratic order $\mathbb{Z}\left[\left(D_{f}+\sqrt{D_{f}}\right) / 2\right]$, or equivalently

$$
t_{D_{f}}=\log \left(\left(u_{D_{f}}+v_{D_{f}} \sqrt{D_{f}}\right) / 2\right)
$$

where $\left(u_{D_{f}}, v_{D_{f}}\right) \in \mathbb{N}^{2}$ is the least solution to $x^{2}-D_{f} y^{2}= \pm 4$. Then, we have

$$
N_{\mathbb{Z}, f}^{\left(D_{4}\right)}(X)=\frac{1}{s_{f} r_{f}} \frac{32 t_{D_{f}}}{9 D_{f}^{3 / 2}} X^{3 / 2}+O_{f}\left(X^{1+\epsilon}\right) \text { for any } \epsilon>0
$$


where

$$
r_{f}=\left\{\begin{array}{l}
2 \quad \text { if } f(x, y) \sim a x^{2}+b x y-a y^{2} \\
\quad \text { or } f(x, y) \sim a x^{2}+b x y+c y^{2} \text { with } a \mid b \\
1 \quad \text { otherwise. }
\end{array}\right.
$$

(d) In all three cases, for any $\epsilon>0$, we have

$$
N_{\mathbb{Z}, f}^{\left(V_{4}\right)}(X)=O_{f, \epsilon}\left(X^{1+\epsilon}\right)
$$

and also

$$
N_{\mathbb{Z}, f}^{\left(C_{4}\right)}(X)= \begin{cases}O_{f, \epsilon}\left(X^{1 / 2+\epsilon}\right) & \text { if }-\Delta(f) \neq \square \\ O_{f}(X) & \text { if }-\Delta(f)=\square .\end{cases}
$$

Notice that the error terms in Theorem 1.2 depend upon $f$. Hence, we are unable to obtain an asymptotic formula for $N_{\mathbb{Z}}^{\dagger}(X)$ by summing over $f \in \mathfrak{F}$. However, there are only three $f \in \mathfrak{F}$ that need to be considered if we restrict to the forms in

$$
V_{\mathbb{Z}}^{\mathrm{sm}, *}=\left\{F \in V_{\mathbb{Z}}^{\mathrm{sm}}: F_{T}=F \text { for some } T \in \mathrm{GL}_{2}(\mathbb{Z}) \backslash\left\{ \pm I_{2 \times 2}\right\}\right\} .
$$

This is because by Proposition 2.1 below, such a matrix $T$ must be of the shape $M_{f}$ or $M_{f} / 2$ up to sign, where $f \in \mathfrak{F}^{*}$. From (1.9), we then deduce that

$$
\begin{aligned}
V_{\mathbb{Z}}^{\mathrm{sm}, *}= & \bigcup_{\substack{f \in \mathfrak{F}^{*} \\
\Delta(f) \in\{-4,1,4\}}}\left\{F \in V_{\mathbb{Z}, f}^{0}: F \text { is irreducible }\right\}, \\
V_{\mathbb{Z}}^{\mathrm{sm}, *, \dagger}= & \bigsqcup_{\substack{f \in \mathfrak{F}^{*} \\
\Delta(f) \in\{-4,1,4\}}}\left\{F \in V_{\mathbb{Z}, f}^{0}: F \text { is irreducible and } \operatorname{Gal}(F) \nsucceq V_{4}\right\} .
\end{aligned}
$$

For $X>0$, let us put

$$
N_{\mathbb{Z}}^{*, \dagger}(X)=\#\left\{[F]: F \in V_{\mathbb{Z}}^{\mathrm{sm}, *, \dagger} \text { such that } H(F) \leq X\right\} .
$$

Then, by (1.8) and the above discussion, we have

$$
N_{\mathbb{Z}}^{*, \dagger}(X)=N_{\mathbb{Z}, f^{(1)}}^{*, \dagger}(X)+N_{\mathbb{Z}, f^{(2)}}^{*, \dagger}(X)+N_{\mathbb{Z}, f^{(3)}}^{*, \dagger}(X),
$$

where we may take

$$
f^{(1)}(x, y)=x^{2}+y^{2}, f^{(2)}(x, y)=x^{2}+x y, f^{(3)}(x, y)=x^{2}+2 x y,
$$

whose discriminants are $-4,1$, and 4 , respectively. It follows that:

Corollary 1.3. We have

$$
N_{\mathbb{Z}}^{*, \dagger}(X)=\frac{1}{9} X^{3 / 2} \log X+O\left(X^{3 / 2}\right) .
$$

Proof. Theorem 1.2 implies that

$$
N_{\mathbb{Z}, f^{(1)}}^{\dagger}(X)=O\left(X^{3 / 2}\right) \text { and } N_{\mathbb{Z}, f^{(i)}}^{\dagger}(X)=\frac{1}{18} X^{3 / 2} \log X+O\left(X^{3 / 2}\right) \text { for } i=2,3
$$

Summing these terms up then yields the claim.

Finally, as a consequence of the proof of Theorem 1.2, we also have:

Theorem 1.4. Let $D=\beta^{2}+4 \alpha^{2}$, where $\alpha, \beta \in \mathbb{N}$ are coprime and $D$ is not a square. Then, the negative Pell's equation $x^{2}-D y^{2}=-4$ has integer solutions if and only if the integral binary quadratic form $\alpha x^{2}+\beta x y-\alpha y^{2}$ is $\mathrm{GL}_{2}(\mathbb{Z})$-equivalent to a form of the shape $a x^{2}+b x y+c y^{2}$ with a dividing $b$. 
We now discuss some potential applications of our Theorem 1.2 and Corollary 1.3.

First, it is natural to ask whether the asymptotic formula (1.3), which was proven using Proposition 5.1, admits a secondary main term. From the arguments in [4], we see that the error term arising from volumes of the lower dimensional projections in Proposition 5.1 is only of order $O\left(X^{3 / 4}\right)$. Thus, possibly $X^{3 / 4}$ is the order of a second main term, but it is dominated by another error term coming from

$$
N_{\mathbb{Z}, \mathrm{BS}}^{*}(X)=\#\left\{[F]: F \in V_{\mathbb{Z}}^{\mathrm{sm}, *} \text { such that } H_{\mathrm{BS}}(F) \leq X\right\} .
$$

In particular, it was shown in [4, Lemma 2.4] that

$$
N_{\mathbb{Z}, \mathrm{BS}}^{*}(X)=O_{\epsilon}\left(X^{3 / 4+\epsilon}\right) \text { for any } \epsilon>0 .
$$

Our Corollary 1.3 removes this obstacle, because

$$
N_{\mathbb{Z}}^{*, \dagger}\left(X^{1 / 3}\right) \leq N_{\mathbb{Z}, \mathrm{BS}}^{*}(X) \leq N_{\mathbb{Z}}^{*, \dagger}\left(10 X^{1 / 3}\right)+O_{\epsilon}\left(X^{1 / 3+\epsilon}\right)
$$

by (1.6) and Theorem $1.2(\mathrm{~d})$, whence we have

$$
N_{\mathbb{Z}, \mathrm{BS}}^{*}(X) \asymp X^{1 / 2} \log X .
$$

This improvement potentially allows one to prove a secondary main term for (1.3) by using similar methods from [5], where it was shown that the counting theorem in [14] for cubic fields has a secondary main term of order $X^{5 / 6}$; this latter fact was proven independently in [23] as well.

Next, integral binary quartic forms are closely related to quartic orders, and maximal irreducible quartic orders may be regarded as quartic fields. More generally, by the construction of Birch-Merriman [7] or Nakagawa [20], any integral binary form $F$ gives rise to a $\mathbb{Z}$-order $Q_{F}$ whose rank is the degree of $F$, where $\mathrm{GL}_{2}(\mathbb{Z})$-equivalence class of $F$ corresponds to isomorphism class of $Q_{F}$. By [15], it is well-known that all cubic orders come from integral binary cubic forms, which enabled the enumeration of cubic orders having a non-trivial automorphism as well as cubic fields by their discriminant; see [6] and [14], respectively. But this is not true for orders of higher rank. Parametrizations of quartic and quintic orders were given by Bhargava in his seminal work [2] and [3]. In [25], Wood further showed that the quartic orders arising from integral binary quartic forms are exactly those having a monogenic cubic resolvent; see [2] for the definition. This implies that the forms in

$$
V_{\mathbb{Z}}^{\mathrm{sm}, \star}=\left\{F \in V_{\mathbb{Z}}^{\mathrm{sm}}: Q_{F} \text { is maximal }\right\}
$$

correspond to quartic $D_{4^{-}}, C_{4^{-}}$, and $V_{4}$-fields whose ring of integers has a monogenic cubic resolvent. In our upcoming paper [24], we shall enumerate $\mathrm{GL}_{2}(\mathbb{Z})$-equivalence classes of forms in $V_{\mathbb{Z}}^{\text {sm, }}$ with respect to a height corresponding to the conductor of fields, as motivated by [1]. In fact, we shall that show that

$$
\text { for all } f \in \mathfrak{F}^{*}: F \in V_{\mathbb{Z}}^{\mathrm{sm}, \star} \cap V_{\mathbb{Z}, f}^{0} \neq \emptyset \text { if and only if } \Delta(f) \in\{-4,1,4\} \text {. }
$$

Thus, our counting theorem in [24] may be regarded as a refinement and an extension of Corollary 1.3 above. 
Last but not least, binary quartic forms are connected to elliptic curves as well. In particular, any integral binary quartic form $F$ gives rise to an elliptic curve

$$
E_{F}: y^{2}=x^{3}-\frac{I(F)}{3} x-\frac{J(F)}{27}
$$

defined over $\mathbb{Q}$. In [4], Bhargava and Shankar applied (1.3) as well as a parametrization of 2-Selmer groups due to Birch and Swinnerton-Dyer to show that the average rank of elliptic curves over $\mathbb{Q}$, when ordered by a naive height analogous to (1.2), is at most $3 / 2$. This result is remarkable in that it is the first to show, unconditional on the BSD-conjecture and the Grand Riemann Hypothesis, boundedness of the average rank of large families of elliptic curves over $\mathbb{Q}$. Conditional bounds were obtained by Brumer [8], Heath-Brown [17], and Young [26] previously. Now, the relations in (1.5) imply that for $F \in V_{\mathbb{Z}}^{\text {sm }} \cap V_{\mathbb{Z}, f}^{0}$ with $f \in \mathfrak{F}^{*}$, we have

$$
E_{F}: y^{2}=\left(x+\frac{L_{f}(F)}{3}\right)\left(x^{2}-\frac{L_{f}(F)}{3} x-\frac{K_{f}(F)}{9}\right),
$$

which has a rational 2-torsion point. Hence, our Theorem 1.2 potentially allows one to study arithmetic properties of elliptic curves with 2 -torsion over $\mathbb{Q}$. Let us remark that unlike a large family of elliptic curves over $\mathbb{Q}$, in the sense of [4, Section 3], the family consisting of those curves with a rational 2-torsion exhibits a rather peculiar behaviour. Indeed, Klagsbrun and Lemke-Oliver [19] proved that the average size of the 2-Selmer groups in this family is unbounded, and they conjectured an asymptotic growth rate. One might be able to obtain such an asymptotic growth rate using our Theorem 1.2 and a sieve that detects local solubility; this line of inquiry is pursued in an upcoming paper due to D. Kane and Z. Klagsbrun.

\section{Characterization of forms with small Galois groups}

2.1. Cremona covariants. Let $F$ be a real binary quartic form with $\Delta(F) \neq 0$. As Cremona defined in [10], we have three quadratic covariants $\mathfrak{C}_{F, \omega}(x, y)$, each of which is associated to a root $\omega$ of $\mathcal{Q}_{F}(x)$; see [27, Subsection 4.2] for the explicit definition. They satisfy the syzygy

$$
\mathfrak{C}_{F, \omega}(x, y)^{2}=\frac{1}{3}\left(F_{4}(x, y)+4 \omega F(x, y)\right),
$$

where $F_{4}$ is the Hessian covariant of $F$ and is given by

$$
\begin{array}{r}
F_{4}(x, y)=3\left(a_{3}^{2}-8 a_{4} a_{2}\right) x^{4}+4\left(a_{3} a_{2}-6 a_{4} a_{1}\right) x^{3} y+2\left(2 a_{2}^{2}-24 a_{4} a_{0}-3 a_{3} a_{1}\right) x^{2} y^{2} \\
+4\left(a_{2} a_{1}-6 a_{3} a_{0}\right) x y^{3}+\left(3 a_{1}^{2}-8 a_{2} a_{0}\right) y^{4} .
\end{array}
$$

We shall label the roots $\omega_{1}(F), \omega_{2}(F), \omega_{3}(F)$ of $\mathcal{Q}_{F}(x)$ such that

$$
\mathfrak{C}_{F, \omega_{i}(F)}(x, y)=\mathfrak{C}_{F, i}(x, y) \text { for all } i=1,2,3,
$$

where $\mathfrak{C}_{F, i}(x, y)$ is defined as in $[27,(4.6)]$. Then, from (2.1) and the explicit expressions for $\mathfrak{C}_{F, \omega}(x, y)$ given in [27], we have the following observations:

(1) For $\omega=\omega_{1}(F)$, the binary quadratic form $\mathfrak{C}_{F, \omega}(x, y)$ has real coefficients.

(2) For $\omega=\omega_{2}(F), \omega_{3}(F)$, we have:

- If $\Delta(F)>0$, then $\lambda_{\omega} \cdot \mathfrak{C}_{F, \omega}(x, y)$ has real coefficients for some $\lambda_{\omega} \in\{1, \sqrt{-1}\}$.

- If $\Delta(F)<0$, then $\lambda \cdot \mathfrak{C}_{F, \omega}(x, y)$ does not have real coefficients for all $\lambda \in \mathbb{C}^{\times}$. 
Also, it is easy to check that

$$
\Delta\left(\mathfrak{C}_{F, \omega_{1}(F)}\right), \Delta\left(\mathfrak{C}_{F, \omega_{3}(F)}\right)>0 \text { and } \Delta\left(\mathfrak{C}_{F, \omega_{2}(F)}\right)<0 .
$$

We shall require the following result by the second-named author in [27].

Proposition 2.1. Let $F$ be a real binary quartic form with $\Delta(F) \neq 0$. Then, a set of representatives for the quotient group

$$
\left\{T \in \mathrm{GL}_{2}(\mathbb{R}): F_{T}=F\right\} /\left\{\lambda \cdot I_{2 \times 2}: \lambda \in \mathbb{R}^{\times}\right\}
$$

is given by

$$
\begin{cases}\left\{I_{2 \times 2}, M_{f}: f \in\left\{\mathfrak{C}_{F, \omega_{1}(F)}, \lambda_{\omega_{2}(F)} \cdot \mathfrak{C}_{F, \omega_{2}(F)}, \lambda_{\omega_{3}(F)} \cdot \mathfrak{C}_{F, \omega_{3}(F)}\right\}\right. & \text { if } \Delta(F)>0, \\ \left\{I_{2 \times 2}, M_{f}: f \in\left\{\mathfrak{C}_{F, \omega_{1}(F)}\right\}\right\} & \text { if } \Delta(F)<0 .\end{cases}
$$

Furthermore, the quadratic forms $\mathfrak{C}_{F, \omega_{1}(F)}(x, y), \mathfrak{C}_{F, \omega_{2}(F)}(x, y)$, and $\mathfrak{C}_{F, \omega_{3}(F)}(x, y)$, are pairwise non-proportional over $\mathbb{C}^{\times}$.

Proof. For the first statement, see [27, Proposition 4.6]. As for the second statement, since $\mathfrak{C}_{F, \omega_{i}(F)}(x, y)$ are covariants, replacing $F$ by a $\mathrm{GL}_{2}(\mathbb{R})$-translate if necessary, we may assume that $F(x, y)=a_{4} x^{4}+a_{2} x^{2} y^{2} \pm a_{4} y^{4}$. In this special case, it is not hard to verify the claim using the explicit expressions for $\mathfrak{C}_{F, \omega_{i}(F)}(x, y)$ in $[27,(4.6)]$.

Let $F$ be a real binary quartic form with $\Delta(F) \neq 0$. Proposition 2.1 implies that for any real binary quadratic form $f$ with $\Delta(f) \neq 0$, we have $F \in V_{\mathbb{R}, f}$ if and only if

$$
f(x, y) \text { is proportional to } \mathfrak{C}_{F, \omega}(x, y) \text { for a root } \omega \text { of } \mathcal{Q}_{F}(x) .
$$

Moreover, this root $\omega$ is unique, and we shall denote it by $\omega_{f}(F)$. This was required in order to define the $L_{f}$ - and $K_{f}$-invariants in (1.4).

\subsection{Proof of Theorem 1.1. The key is the following lemma.}

Lemma 2.2. Let $F$ be an integral binary quartic form with $\Delta(F) \neq 0$ and let $\omega$ be a root of $\mathcal{Q}_{F}(x)$. Then, the quadratic form $\mathfrak{C}_{F, \omega}(x, y)$ is proportional over $\mathbb{C}^{\times}$to a form with integer coefficients if and only if $\omega \in \mathbb{Z}$.

Proof. If $\omega \in \mathbb{Z}$, then we easily see from $(2.1)$ that $\lambda \cdot \mathfrak{C}_{F, \omega}(x, y)$ has integer coefficients for some $\lambda \in \mathbb{C}^{\times}$. Conversely, if $\lambda \cdot \mathfrak{C}_{F, \omega}(x, y)$ has integer coefficients for some $\lambda \in \mathbb{C}^{\times}$, then consider the action of an element $\sigma \in \operatorname{Gal}(\overline{\mathbb{Q}} / \mathbb{Q})$, where $\overline{\mathbb{Q}}$ is an algebraic closure of $\mathbb{Q}$. It is clear from the definition of $\mathfrak{C}_{F, \omega}(x, y)$ that $\lambda \in \overline{\mathbb{Q}}$. From $(2.1)$, we have

$$
\frac{4}{3}(\omega-\sigma(\omega)) F(x, y)=\mathfrak{C}_{F, \omega}(x, y)^{2}-\sigma\left(\mathfrak{C}_{F, \omega}(x, y)^{2}\right)=\left(1-\frac{\lambda^{2}}{\sigma(\lambda)^{2}}\right) \mathfrak{C}_{F, \omega}(x, y)^{2},
$$

and this last binary quartic form has zero discriminant. This shows that $\omega-\sigma(\omega)=0$ for all $\sigma \in \operatorname{Gal}(\overline{\mathbb{Q}} / \mathbb{Q})$. Thus, we have $\omega \in \mathbb{Q}$, and so $\omega \in \mathbb{Z}$ since $\mathcal{Q}_{F}(x)$ is monic.

The first claim in Theorem 1.1 now follows from Proposition 2.1, Lemma 2.2, and (2.3). Note that $\Delta(F)=27^{2} \Delta\left(\mathcal{Q}_{F}\right)$, which means that $\mathcal{Q}_{F}(x)$ has three integer roots if and only if $\mathcal{Q}_{F}(x)$ is reducible and $\Delta(F)=\square$. The second claim then follows from this fact and (2.2). 


\section{BASIC PROPERTIES OF FORMS IN $V_{\mathbb{R}, f}$ OF NON-ZERO DISCRIMINANT}

Throughout this section, let $f(x, y)=\alpha x^{2}+\beta x y+\gamma y^{2}$ be a real binary quadratic form with $\Delta(f) \neq 0$. It is not hard to check, by a direct calculation, that

$$
V_{\mathbb{R}, f}=\left\{\begin{array}{c}
A x^{4}+B x^{3} y+C x^{2} y^{2}+\left(\frac{4 \beta \gamma A-\left(\beta^{2}+2 \alpha \gamma\right) B+2 \alpha \beta C}{2 \alpha^{2}}\right) x y^{3} \\
+\left(\frac{4 \gamma\left(\beta^{2}+2 \alpha \gamma\right) A-\beta\left(\beta^{2}+4 \alpha \gamma\right) B+2 \alpha \beta^{2} C}{8 \alpha^{3}}\right) y^{4}: A, B, C \in \mathbb{R}
\end{array}\right\}
$$

if $\alpha \neq 0$, and similarly that

$$
V_{\mathbb{R}, f}=\left\{\begin{array}{c}
A x^{4}+\left(\frac{\gamma\left(4 \beta^{2}+8 \alpha \gamma\right) A+2 \alpha \beta^{2} B-8 \alpha^{3} C}{\beta\left(\beta^{2}+4 \alpha \gamma\right)}\right) x^{3} y+B x^{2} y^{2} \\
-\left(\frac{8 \gamma^{3} A-2 \beta^{2} \gamma B-\alpha\left(4 \beta^{2}+8 \alpha \gamma\right) C}{\beta\left(\beta^{2}+4 \alpha \gamma\right)}\right) x y^{3}+C y^{4}: A, B, C \in \mathbb{R}
\end{array}\right\}
$$

if $\beta, \beta^{2}+4 \alpha \gamma \neq 0$. Below, we shall give some basic properties of $V_{\mathbb{R}, f}^{0}$ and $V_{\mathbb{Z}, f}^{0}$.

3.1. The two new invariants. Recall the definitions of the $L_{f^{-}}$and $K_{f}$-invariants given in (1.4). First, we shall show that they are indeed invariants under the twisted action of $\mathrm{GL}_{2}(\mathbb{R})$ in the following sense.

Proposition 3.1. For all $F \in V_{\mathbb{R}, f}^{0}$ and $T \in \mathrm{GL}_{2}(\mathbb{R})$, we have

$$
L_{f_{T}}\left(F_{T}\right)=L_{f}(F) \text { and } K_{f_{T}}\left(F_{T}\right)=K_{f}(F) .
$$

Proof. Notice that $\mathcal{Q}_{F}(x)=\mathcal{Q}_{F_{T}}(x)$. For any root $\omega$ of $\mathcal{Q}_{F}(x)$, because $\mathfrak{C}_{F, \omega}(x, y)$ is a covariant up to sign by $(2.1)$, if $\mathfrak{C}_{F, \omega}(x, y)$ is proportional to $f(x, y)$, then $\mathfrak{C}_{F_{T}, \omega}(x, y)$ is proportional to $f_{T}(x, y)$. It then follows from the definition that $L_{f_{T}}\left(F_{T}\right)=L_{f}(F)$. Since $I\left(F_{T}\right)=I(F)$, we also have $K_{f_{T}}\left(F_{T}\right)=K_{f}(F)$ by the first equality in (1.5).

We shall give explicit formulae for $L_{f}(-)$ and $K_{f}(-)$ in two special cases.

Proposition 3.2. The following holds.

(a) Assume that $\alpha \neq 0$. Then, for all $F \in V_{\mathbb{R}, f}^{0}$ as in (3.1), we have

$$
\begin{aligned}
L_{f}(F)= & -(12 \gamma A-3 \beta B+2 \alpha C) /(2 \alpha), \\
K_{f}(F)= & \left(72 \beta^{2} \gamma A^{2}+9 \alpha\left(\beta^{2}+4 \alpha \gamma\right) B^{2}+8 \alpha^{3} C^{2}\right. \\
& \left.\quad-18 \beta\left(\beta^{2}+4 \alpha \gamma\right) A B+12 \alpha\left(3 \beta^{2}-4 \alpha \gamma\right) A C-24 \alpha^{2} \beta B C\right) /\left(4 \alpha^{3}\right) .
\end{aligned}
$$

Moreover, we have

$$
\frac{4\left(L_{f}(F)^{2}+4 K_{f}(F)\right)}{9}=\frac{L_{f, 1}(F)^{2}-\Delta(f) L_{f, 2}(F)^{2}}{\alpha^{4}}
$$

where

$$
L_{f, 1}(F)=4\left(\beta^{2}-\alpha \gamma\right) A-3 \alpha \beta B+2 \alpha^{2} C \text { and } L_{f, 2}(F)=2(2 \beta A-\alpha B) .
$$

(b) Assume that $\gamma=0$. Then, for all $F \in V_{\mathbb{R}, f}^{0}$ as in (3.2), we have

$$
\begin{aligned}
& L_{f}(F)=\left(2 \beta^{2} B-12 \alpha^{2} C\right) / \beta^{2}, \\
& K_{f}(F)=\left(-\beta^{4} B^{2}+144 \alpha^{4} C^{2}+36 \beta^{4} A C-24 \alpha^{2} \beta^{2} B C\right) / \beta^{4} .
\end{aligned}
$$


Moreover, we have

$$
\frac{4\left(L_{f}(F)^{2}+4 K_{f}(F)\right)}{9}=\frac{8 C}{\beta^{2}}\left(8 \beta^{2} A-8 \alpha^{2} B+\frac{40 \alpha^{4}}{\beta^{2}} C\right) .
$$

Proof. This may be verified by explicit computation.

We shall also need the following observation.

Proposition 3.3. Assume that $f$ is integral. Then, for all $F \in V_{\mathbb{Z}, f}^{0}$, we have

$$
L_{f}(F), K_{f}(F),\left(L_{f}(F)^{2}+4 K_{f}(F)\right) / 9,\left(2 L_{f}(F)^{2}-K_{f}(F)\right) / 9 \in \mathbb{Z} .
$$

Moreover, when $f$ is primitive in addition, we have

$$
4\left(2 L_{f}(F)^{2}-K_{f}(F)\right) /(9 \Delta(f)) \in \mathbb{Z} .
$$

Proof. We have $L_{f}(F) \in \mathbb{Z}$ by Lemma 2.2. Since $I(F) \in \mathbb{Z}$, we deduce from the first equality in (1.5) that $K_{f}(F) \in \mathbb{Z}$ holds as well. Observe that

$$
\begin{aligned}
I(F)+K_{f}(F) & =\left(L_{f}(F)^{2}+4 K_{f}(F)\right) / 3, \\
2 I(F)-K_{f}(F) & =\left(2 L_{f}(F)^{2}-K_{f}(F)\right) / 3,
\end{aligned}
$$

both of which are integers. Since $\Delta(F) \in \mathbb{Z}$, we deduce from (1.7) that at least one of the above expressions is divisible by 3 . But again by (1.5), we have

$$
3 I(F)=\left(L_{f}(F)^{2}+4 K_{f}(F)\right) / 3+\left(2 L_{f}(F)^{2}-K_{f}(F)\right) / 3,
$$

so in fact both expressions are divisible by 3 . This proves the first claim.

Next, assume that $f$ is primitive in addition. In view of Proposition 3.1, by applying a $\mathrm{GL}_{2}(\mathbb{Z})$-action on $f$ if necessary, we may assume that $\alpha \neq 0$ and that $\alpha$ is coprime to $\Delta(f)$. Using Proposition 3.2 (a), we then compute that

$$
\frac{4\left(2 L_{f}(F)^{2}-K_{f}(F)\right)}{9}=\Delta(f)\left(\frac{\alpha\left(B^{2}-4 A C\right)+2 A(\beta B-4 \gamma A)}{\alpha^{3}}\right) .
$$

This expression is an integer by the first claim, and hence must be divisible by $\Delta(f)$, because $\alpha$ is taken to be coprime to $\Delta(f)$. This proves the second claim.

3.2. Determinants of the two lattices. In this subsection, assume that $f$ is integral and primitive. Let $\Lambda_{f, 1}$ and $\Lambda_{f, 2}$ denote the lattices defined in (1.10). Below, we shall compute their determinants in terms of the number $s_{f}$ as in Theorem 1.2.

Proposition 3.4. We have $\operatorname{det}\left(\Lambda_{f, 1}\right)=s_{f}|\alpha|^{3}$ and $\operatorname{det}\left(\Lambda_{f, 2}\right)=s_{f}\left|\beta\left(\beta^{2}+4 \alpha \gamma\right)\right| / 8$.

Proof. Observe that the linear transformation defined by the matrix

$$
\left(\begin{array}{ccc}
1 & 0 & 0 \\
0 & 0 & 1 \\
* & -\mathcal{B} & *
\end{array}\right), \text { where } \mathcal{B}=\frac{\beta\left(\beta^{2}+4 \alpha \gamma\right)}{8 \alpha^{3}}
$$

has determinant $\mathcal{B}$, and it sends $\Lambda_{f, 1}$ to $\Lambda_{f, 2}$. Thus, it suffices to prove the first claim. Recall from (3.1) that $\Lambda_{f, 1}$ is the set of tuples $(A, B, C) \in \mathbb{Z}^{3}$ satisfying

$$
\begin{aligned}
4 \beta \gamma A-\left(\beta^{2}+2 \alpha \gamma\right) B+2 \alpha \beta C & \equiv 0\left(\bmod 2 \alpha^{2}\right), \\
4 \gamma\left(\beta^{2}+2 \alpha \gamma\right) A-\beta\left(\beta^{2}+4 \alpha \gamma\right) B+2 \alpha \beta^{2} C & \equiv 0\left(\bmod 8 \alpha^{3}\right) .
\end{aligned}
$$


If $\beta \gamma=0$, then it is easy to check that $\operatorname{det}\left(\Lambda_{f, 1}\right)=s_{f}|\alpha|^{3}$. If $\beta \gamma \neq 0$, then we shall use the fact that

$$
\operatorname{det}\left(\Lambda_{f, 1}\right)=\prod_{p} \operatorname{det}\left(\Lambda_{f, 1}^{(p)}\right)=\prod_{p \mid 2 \alpha} \operatorname{det}\left(\Lambda_{f, 1}^{(p)}\right), \text { where } \Lambda_{f, 1}^{(p)}=\mathbb{Z}_{p} \otimes_{\mathbb{Z}} \Lambda_{f, 1},
$$

and so $\operatorname{det}\left(\Lambda_{f, 1}\right)=s_{f}|\alpha|^{3}$ indeed holds by Lemma 3.5 below.

Lemma 3.5. Let $p$ be a prime dividing $2 \alpha$ and let $p^{k} \| \alpha$. Then, we have

$$
\operatorname{det}\left(\Lambda_{f, 1}^{(p)}\right)=s_{f}^{\epsilon_{p}} p^{3 k}, \text { where } \epsilon_{p}= \begin{cases}1 & \text { if } p=2, \\ 0 & \text { if } p \geq 3 .\end{cases}
$$

Proof. For brevity, write

$$
\alpha=p^{k} a \text { and } \beta=p^{\ell} b, \text { where } k, \ell, a, b \in \mathbb{Z} \text { with } k, \ell \geq 0 \text { and } p \nmid a, b .
$$

Then, the claim may be restated as

$$
\operatorname{det}\left(\Lambda_{f, 1}^{(p)}\right)= \begin{cases}p^{3 k+3 \epsilon_{p}} & \text { if } \ell=0 \\ p^{3 k} & \text { if } \ell \geq 1\end{cases}
$$

By definition, the lattice $\Lambda_{f, 1}^{(p)}$ is the set $(A, B, C) \in \mathbb{Z}_{p}^{3}$ of tuples satisfying

$$
\mathcal{T}_{1}(A, B, C) \equiv 0\left(\bmod p^{2 k+\epsilon_{p}}\right) \text { and } \mathcal{T}_{2}(A, B, C) \equiv 0\left(\bmod p^{3 k+3 \epsilon_{p}}\right),
$$

where

$$
\begin{aligned}
& \mathcal{T}_{1}(A, B, C)=p^{\ell} b\left(4 \gamma A-p^{\ell} b B\right)-2 p^{k} a \gamma B+2 p^{k+\ell} a b C, \\
& \mathcal{T}_{2}(A, B, C)=\left(p^{2 \ell} b^{2}+4 p^{k} a \gamma\right)\left(4 \gamma A-p^{\ell} b B\right)-8 p^{k} a \gamma^{2} A+2 p^{k+2 \ell} a b^{2} C .
\end{aligned}
$$

Observe that we have the relation

$$
\mathcal{T}_{2}(A, B, C)-p^{\ell} b \mathcal{T}_{1}(A, B, C)=2 p^{k} a \gamma\left(4 \gamma A-p^{\ell} b B\right) .
$$

For $\ell=0$, we deduce from (3.3) that $\Lambda_{f, 1}^{(p)}$ is defined solely by

$$
\mathcal{T}_{2}(A, B, C) \equiv 0\left(\bmod p^{3 k+3 \epsilon_{p}}\right) .
$$

For $\ell \geq 1$ and $\ell \geq k+2 \epsilon_{p}$, it is easy to see that $\Lambda_{f, 1}^{(p)}$ is in fact defined by

$$
A \equiv 0\left(\bmod p^{2 k}\right) \text { and } B \equiv 0\left(\bmod p^{k}\right) \text {. }
$$

For $\ell \geq 1$ and $\ell \leq k+\epsilon_{p}$, we shall first show that $\Lambda_{f, 1}^{(p)}$ is also defined by

$$
\begin{cases}A \equiv 0 & \left(\bmod p^{2 \ell-2 \epsilon_{p}}\right), \\ B \equiv 0 & \left(\bmod p^{\ell-\epsilon_{p}}\right), \\ \left(4 \gamma A-p^{\ell} b B\right) / p^{2 \ell-\epsilon_{p}} \equiv 0 & \left(\bmod p^{k-\ell+\epsilon_{p}}\right) \\ \mathcal{T}_{2}(A, B, C) / p^{k+2 \ell+\epsilon_{p}} \equiv 0 & \left(\bmod p^{2 k-2 \ell+2 \epsilon_{p}}\right) .\end{cases}
$$

If (3.4) is satisfied, then from (3.3), it is easy to see that $(A, B, C) \in \Lambda_{f, 1}^{(p)}$. Conversely, if $(A, B, C) \in \Lambda_{f, 1}^{(p)}$, then the assumption $\ell \leq k+\epsilon_{p}$ implies that

$$
\mathcal{T}_{1}(A, B, C) \equiv 0\left(\bmod p^{k+\ell}\right) \text { and } \mathcal{T}_{2}(A, B, C) \equiv 0\left(\bmod p^{k+2 \ell+\epsilon_{p}}\right),
$$

while reducing (3.3) $\bmod p^{2 k+\ell+\epsilon_{p}}$ also yields

$$
4 \gamma A-p^{\ell} b B \equiv 0\left(\bmod p^{k+\ell}\right) \text {. }
$$


From these three congruence equations, it follows that (3.4) is indeed satisfied. In all cases, we then see that $\operatorname{det}\left(\Lambda_{f, 1}^{(p)}\right)$ is as claimed.

3.3. Forms with abelian Galois groups. In this subsection, assume that $f$ is integral. Consider an irreducible form $F \in V_{\mathbb{Z}, f}^{0}$. By Theorem 1.1, we have $\operatorname{Gal}(F) \simeq D_{4}$, $C_{4}$, or $V_{4}$. To distinguish among these three possibilities, note that the cubic resolvent polynomial of $F$, defined by

$$
R_{F}(x)=a_{4}^{3} X^{3}-a_{4}^{2} a_{2} X^{2}+a_{4}\left(a_{3} a_{1}-4 a_{4} a_{0}\right) X-\left(a_{3}^{2} a_{0}+a_{4} a_{1}^{2}-4 a_{4} a_{2} a_{0}\right)
$$

when $F$ has the shape (1.1), is reducible since $\operatorname{Gal}(F)$ is small. Also, it has a unique root $r_{F} \in \mathbb{Q}$ precisely when $\Delta(F) \neq \square$, in which case we define

$$
\theta_{1}(F)=\left(a_{3}^{2}-4 a_{4}\left(a_{2}-r_{F} a_{4}\right)\right) \Delta(F) \text { and } \theta_{2}(F)=a_{4}\left(r_{F}^{2} a_{4}-4 a_{0}\right) \Delta(F) .
$$

Then, we have the well-known criterion

$$
\begin{aligned}
\operatorname{Gal}(F) \simeq V_{4} & \Longleftrightarrow \Delta(F)=\square, \\
\operatorname{Gal}(F) \simeq C_{4} & \Longleftrightarrow \Delta(F) \neq \square \text { and } \theta_{1}(F), \theta_{2}(F)=\square \text { in } \mathbb{Q} .
\end{aligned}
$$

See [9] for example. We then deduce that:

Proposition 3.6. Let $F \in V_{\mathbb{Z}, f}^{0}$ be an irreducible form. Then, we have

$$
\operatorname{Gal}(F) \simeq V_{4} \Longleftrightarrow L_{f}(F)^{2}+4 K_{f}(F)=\square,
$$

as well as

$$
\operatorname{Gal}(F) \simeq C_{4} \Longleftrightarrow\left\{\begin{array}{l}
L_{f}(F)^{2}+4 K_{f}(F) \neq \square \\
\left(L_{f}(F)^{2}+4 K_{f}(F)\right)\left(2 L_{f}(F)^{2}-K_{f}(F)\right) / \Delta(f)=\square
\end{array}\right.
$$

Proof. Observe that by (1.7), we have

$$
\Delta(F)=\square \text { if and only if } L_{f}(F)^{2}+4 K_{f}(F)=\square .
$$

The first claim is then clear. Next, suppose that $\Delta(F) \neq \square$. By Proposition 3.1, we may assume that $\alpha \neq 0$. For $F$ in the shape as in (3.1), a direct computation yields

$$
r_{F}=(-4 \gamma A+\beta B) /(2 \alpha A) .
$$

Using Proposition 3.2 (a), we further compute that

$$
\begin{aligned}
& \theta_{1}(F)=4 \alpha^{2}\left(2 L_{f}(F)^{2}-K_{f}(F)\right) \Delta(F) /(9 \Delta(f)), \\
& \theta_{2}(F)=\beta^{2}\left(2 L_{f}(F)^{2}-K_{f}(F)\right) \Delta(F) /(9 \Delta(f)) .
\end{aligned}
$$

By (1.7) and the criterion above, it follows that $\theta_{1}(F), \theta_{2}(F)$ are squares if and only if $\left(L_{f}(F)^{2}+4 K_{f}(F)\right)\left(2 L_{f}(F)^{2}-K_{f}(F)\right) / \Delta(f)$ is a square, as desired.

3.4. Reducible forms. In this subsection, assume that $f$ is integral. We shall study the reducible forms in $V_{\mathbb{Z}, f}^{0}$. Let us first make a definition and an observation.

Definition 3.7. Let $F \in V_{\mathbb{Z}, f}^{0}$ be a reducible form.

(1) We say that $F$ is of type 1 if $F=m \cdot p p_{M_{f}}$ for some $m \in \mathbb{Q}^{\times}$and integral binary quadratic form $p$.

(2) We say that $F$ is of type 2 if $F=p q$ for some integral binary quadratic forms $p$ and $q$ satisfying $p_{M_{f}}=-p$ and $q_{M_{f}}=-q$. 
Lemma 3.8. For all reducible forms $F \in V_{\mathbb{Z}, f}^{0}$ of type 1 , we have

$$
L_{f}(F)^{2}+4 K_{f}(F)=\square .
$$

Proof. This may be verified by a direct computation.

Below, we shall show that the two reducibility types in Definition 3.7 are in fact the only possibilities. We shall require two further lemmas.

Lemma 3.9. Let $\ell(x, y)=\ell_{1} x+\ell_{0} y$ be a non-zero complex binary linear form, and suppose that $\ell_{M_{f}}=\lambda \cdot \ell$ for some $\lambda \in \mathbb{C}^{\times}$. Then, we have $\lambda= \pm \sqrt{-1}$, with

$$
\lambda= \begin{cases}-\sqrt{-1} & \text { if and only if } \ell_{0}=(\beta+\sqrt{\Delta(f)}) \ell_{1} /(2 \alpha), \\ \sqrt{-1} & \text { if and only if } \ell_{0}=(\beta-\sqrt{\Delta(f)}) \ell_{1} /(2 \alpha),\end{cases}
$$

in the case that $\alpha \neq 0$.

Proof. The hypothesis implies that

$$
\frac{1}{\sqrt{-\Delta(f)}}\left(\begin{array}{cc}
\beta & -2 \alpha \\
2 \gamma & -\beta
\end{array}\right)\left(\begin{array}{l}
\ell_{1} \\
\ell_{0}
\end{array}\right)=\lambda\left(\begin{array}{l}
\ell_{1} \\
\ell_{0}
\end{array}\right) \text {. }
$$

Then, by computing the eigenvalues and eigenspaces of the $2 \times 2$ matrix above, we see that the claim holds.

Lemma 3.10. Let $p(x, y)=p_{2} x^{2}+p_{1} x y+p_{0} y^{2}$ be a non-zero complex binary quadratic form, and suppose that $p_{M_{f}}=\lambda \cdot p$ for some $\lambda \in \mathbb{C}^{\times}$. Then, we have $\lambda= \pm 1$, with

$$
\lambda= \begin{cases}-1 & \text { if and only if } p_{0}=\left(\beta p_{1}-2 \gamma p_{2}\right) /(2 \alpha), \\ 1 & \text { if and only if } p=\left(p_{2} / \alpha\right) f,\end{cases}
$$

in the case that $\alpha \neq 0$.

Proof. The hypothesis implies that

$$
\frac{1}{-\Delta(f)}\left(\begin{array}{ccc}
\beta^{2} & -2 \alpha & 4 \alpha^{2} \\
4 \beta \gamma & -\left(\beta^{2}+4 \alpha \gamma\right) & 4 \alpha \beta \\
4 \gamma^{2} & -2 \beta \gamma & \beta^{2}
\end{array}\right)\left(\begin{array}{l}
p_{2} \\
p_{1} \\
p_{0}
\end{array}\right)=\lambda\left(\begin{array}{l}
p_{2} \\
p_{1} \\
p_{0}
\end{array}\right) .
$$

Then, by computing the eigenvalues and eigenspaces of the $3 \times 3$ matrix above, it is not hard to check that the claim holds.

Proposition 3.11. Any reducible form $F \in V_{\mathbb{Z}, f}^{0}$ is either of type 1 or of type 2 .

Proof. Write $F=g^{(1)} g^{(2)} g^{(3)} g^{(4)}$, where the $g^{(k)}$ are complex binary linear forms, and are pairwise non-proportional because $\Delta(F) \neq 0$. Since $F$ is reducible, by renumbering if necessary, we may assume that

$$
\begin{cases}g^{(1)}, g^{(2)} g^{(3)} g^{(4)} & \text { when } F \text { has exactly one rational linear factor, } \\ g^{(1)}, g^{(2)}, g^{(3)} g^{(4)} & \text { when } F \text { has exactly two rational linear factors, } \\ g^{(1)} g^{(2)}, g^{(3)} g^{(4)} & \text { when } F \text { has no rational linear factor, } \\ g^{(1)}, g^{(2)}, g^{(3)}, g^{(4)} & \text { when } F \text { has four rational linear factors, }\end{cases}
$$

have integer coefficients and are irreducible. We have $M_{f}^{2}=\Delta(f) \cdot I_{2 \times 2}$ and $F_{M_{f}}=F$ by definition. Hence, up to scaling, the matrix $M_{f}$ acts on the $g^{(k)}$ via a permutation 
$\sigma$ on four letters of order dividing two. This has two consequences.

By (1.8), without loss of generality, we may assume that $\alpha \neq 0$. First, the form $F$ cannot have exactly one rational linear factor, for otherwise

$$
\sigma(1)=1 \text { and } \sigma\left(k_{0}\right)=k_{0} \text { for at least one } k_{0} \in\{2,3,4\} .
$$

From Lemma 3.9, it would follow that $\Delta(f)$ is a square and that $g^{\left(k_{0}\right)}$ is proportional to a form with integer coefficients, which is a contradiction. Second, when $F$ has four rational linear factors, by further renumbering if necessary, we may assume that

$$
\sigma \in\{(1),(12),(12)(34)\} .
$$

Now, in all three of the possible cases for the factorization of $F$, define

$$
p=g^{(1)} g^{(2)} \text { and } q=g^{(3)} g^{(4)},
$$

which are integral binary quadratic forms by definition. We then deduce that

$$
\left(p_{M_{f}}, q_{M_{f}}\right)=\left(\lambda \cdot q, \lambda^{-1} \cdot p\right) \text { or }\left(p_{M_{f}}, q_{M_{f}}\right)=\left(\lambda \cdot p, \lambda^{-1} \cdot q\right)
$$

for some $\lambda \in \mathbb{Q}^{\times}$. In the former case, it is clear that $F$ is of type 1 . In the latter case, we have $\lambda=-1$ by Lemma 3.10 and the fact that $\Delta(F) \neq 0$, so $F$ is of type 2 .

\section{Parametrizing FORMs in $V_{\mathbb{R}, f}$ OF NON-ZERO Discriminant}

Throughout this section, let $f(x, y)=\alpha x^{2}+\beta x y+\gamma y^{2}$ be a real binary quadratic form with $\Delta(f) \neq 0$ and $\alpha>0$. We shall give an alternative parametrization of $V_{\mathbb{R}, f}^{0}$, different from (3.1) and (3.2), in terms of the regions

$$
\begin{aligned}
& \Omega^{0}=\left\{(L, K) \in \mathbb{R}^{2} \mid L^{2}+4 K \neq 0 \text { and } 2 L^{2}-K \neq 0\right\}, \\
& \Omega^{+}=\left\{(L, K) \in \mathbb{R}^{2} \mid L^{2}+4 K>0 \text { and } 2 L^{2}-K \neq 0\right\}, \\
& \Omega^{-}=\left\{(L, K) \in \mathbb{R}^{2} \mid L^{2}+4 K<0 \text { and } 2 L^{2}-K>0\right\},
\end{aligned}
$$

corresponding to the $L_{f}$ and $K_{f}$-invariants, as well as a parameter $t \in \mathbb{R}$ arising from the orthogonal group of $f$, defined by

$$
O_{f}(\mathbb{R})=\left\{T \in \mathrm{GL}_{2}(\mathbb{R}): \operatorname{det}(T)= \pm 1 \text { and } f_{T}= \pm f\right\} .
$$

Note that by (1.7), for any $F \in V_{\mathbb{R}, f}^{0}$, we have

$$
\begin{aligned}
& \left(L_{f}(F), K_{f}(F)\right) \in \Omega^{+} \Longleftrightarrow \Delta(F)>0, \\
& \left(L_{f}(F), K_{f}(F)\right) \in \Omega^{-} \Longleftrightarrow \Delta(F)<0 .
\end{aligned}
$$

First, we shall show that it suffices to consider $x^{2}+y^{2}$ and $x^{2}-y^{2}$. It shall be helpful to recall (1.8) as well as the isomorphisms $\Theta_{1}$ and $\Theta_{2}$ defined in Subsection 1.1.

Lemma 4.1. Define a matrix

$$
T_{f}=\left(\begin{array}{cc}
\delta_{f}^{-1 / 4} & 0 \\
0 & \delta_{f}^{1 / 4}
\end{array}\right) \cdot \frac{1}{2 \sqrt{\alpha}}\left(\begin{array}{cc}
2 \alpha & \beta \\
0 & 2
\end{array}\right) \text {, where } \delta_{f}=\frac{|\Delta(f)|}{4}
$$

Then, we have a well-defined bijective linear map

$$
\begin{cases}\Psi_{f}: V_{\mathbb{R}, x^{2}+y^{2}} \longrightarrow V_{\mathbb{R}, f} ; & \Psi_{f}(F)=F_{T_{f}} \quad \text { if } f \text { is positive definite, } \\ \Psi_{f}: V_{\mathbb{R}, x^{2}-y^{2}} \longrightarrow V_{\mathbb{R}, f} ; & \Psi_{f}(F)=F_{T_{f}} \quad \text { if } f \text { is indefinite, }\end{cases}
$$

and we have $\operatorname{det}\left(\Psi_{f}\right)=8 \alpha^{3}|\Delta(f)|^{-3 / 2}$. 
Proof. The first claim holds by (1.8) and the fact

$$
\delta_{f}^{-1 / 2} \cdot f= \begin{cases}\left(x^{2}+y^{2}\right)_{T_{f}} & \text { if } f \text { is positive definite, } \\ \left(x^{2}-y^{2}\right)_{T_{f}} & \text { if } f \text { is indefinite. }\end{cases}
$$

Identifying $V_{\mathbb{R}, x^{2} \pm y^{2}}$ and $V_{\mathbb{R}, f}$ with $\mathbb{R}^{3}$ via $\Theta_{1}$, we see from (3.1) that

$$
\Psi_{f}:\left(\begin{array}{l}
a_{4} \\
a_{3} \\
a_{2}
\end{array}\right) \mapsto\left(\begin{array}{ccc}
\frac{\alpha^{2}}{\delta_{f}} & 0 & 0 \\
\frac{2 \alpha \beta}{\delta_{f}} & \frac{\alpha}{\sqrt{\delta_{f}}} & 0 \\
\frac{3 \beta^{2}}{2 \delta_{f}} & \frac{3 \beta}{2 \sqrt{\delta_{f}}} & 1
\end{array}\right)\left(\begin{array}{l}
a_{4} \\
a_{3} \\
a_{2}
\end{array}\right),
$$

from which the second claim follows.

In the subsequent subsections, we shall prove the following propositions.

Proposition 4.2. There exists an explicit bijection

$$
\Phi: \Omega^{+} \times[-\pi / 4, \pi / 4) \longrightarrow V_{\mathbb{R}, x^{2}+y^{2}}^{0},
$$

defined as in (4.4), such that

(a) we have $L_{x^{2}+y^{2}}(\Phi(L, K, t))=L$ and $K_{x^{2}+y^{2}}(\Phi(L, K, t))=K$,

(b) the Jacobian matrix of $\Theta_{1} \circ \Phi$ has determinant $-1 / 18$.

Proposition 4.3. There exist explicit injections

$$
\Phi^{(1)}, \Phi^{(2)}: \Omega^{+} \times \mathbb{R} \longrightarrow V_{\mathbb{R}, x^{2}-y^{2}}^{0} \text { and } \Phi^{(3)}, \Phi^{(4)}: \Omega^{-} \times \mathbb{R} \longrightarrow V_{\mathbb{R}, x^{2}-y^{2}}^{0},
$$

defined as in (4.6), with

$$
V_{\mathbb{R}, x^{2}-y^{2}}^{0}=\Phi^{(1)}\left(\Omega^{+} \times \mathbb{R}\right) \sqcup \Phi^{(2)}\left(\Omega^{+} \times \mathbb{R}\right) \sqcup \Phi^{(3)}\left(\Omega^{-} \times \mathbb{R}\right) \sqcup \Phi^{(4)}\left(\Omega^{-} \times \mathbb{R}\right)
$$

such that

(a) we have $L_{x^{2}-y^{2}}\left(\Phi^{(i)}(L, K, t)\right)=L$ and $K_{x^{2}-y^{2}}\left(\Phi^{(i)}(L, K, t)\right)=K$,

(b) the Jacobian matrix of $\Theta_{1} \circ \Phi^{(i)}$ has determinant $-1 / 18$,

for all $i=1,2,3,4$.

In view of (1.11), we shall give another parametrization of $V_{\mathbb{R}, f}$ when $\gamma=0$, which does not require reducing to the form $x^{2}-y^{2}$ via Lemma 4.1.

Proposition 4.4. Suppose that $\gamma=0$. Then, there exist explicit injections

$$
\Phi_{f}^{(1)}, \Phi_{f}^{(2)}: \Omega^{0} \times \mathbb{R} \longrightarrow V_{\mathbb{R}, f}^{0},
$$

defined as in (4.9), with

$$
V_{\mathbb{R}, f}^{0}=\Phi_{f}^{(1)}\left(\Omega^{0} \times \mathbb{R}\right) \sqcup \Phi_{f}^{(2)}\left(\Omega^{0} \times \mathbb{R}\right)
$$

such that

(a) we have $L_{f}\left(\Phi^{(i)}(L, K, t)\right)=L$ and $K_{f}\left(\Phi^{(i)}(L, K, t)\right)=K$,

(b) the Jacobian matrix of $\Theta_{2} \circ \Phi_{f}^{(i)}$ has determinant $-1 / 18$, for both $i=1,2$.

For $t \in \mathbb{R}$, we shall use the notation

$$
T^{+}(t)=\left(\begin{array}{cc}
\cos t & \sin t \\
-\sin t & \cos t
\end{array}\right) \text { and } T^{-}(t)=\left(\begin{array}{cc}
\cosh t & \sinh t \\
\sinh t & \cosh t
\end{array}\right),
$$

which is an element of $O_{x^{2}+y^{2}}(\mathbb{R})$ and $O_{x^{2}-y^{2}}(\mathbb{R})$, respectively. 


\subsection{Positive definite case. Define}

$$
\Phi: \Omega^{+} \times[-\pi / 4, \pi / 4) \longrightarrow V_{\mathbb{R}, x^{2}+y^{2}}^{0} ; \quad \Phi(L, K, t)=\left(F_{(L, K)}\right)_{T^{+}(t)},
$$

where

$$
F_{(L, K)}(x, y)=\frac{-3 L+\sqrt{L^{2}+4 K}}{24} x^{4}+\frac{-L-\sqrt{L^{2}+4 K}}{4} x^{2} y^{2}+\frac{-3 L+\sqrt{L^{2}+4 K}}{24} y^{4} .
$$

The image of $\Phi$ lies in $V_{\mathbb{R}, x^{2}+y^{2}}$ by (3.1) and (1.8). Using Propositions 3.1 and 3.2 (a), it is easy to check that Proposition 4.2 (a) holds.

Now, by (3.1), an arbitrary $F \in V_{\mathbb{R}, x^{2}+y^{2}}^{0}$ has the shape

$$
F(x, y)=a_{4} x^{4}+a_{3} x^{3} y+a_{2} x^{2} y^{2}-a_{3} x y^{3}+a_{4} y^{4} .
$$

Write $L=L_{x^{2}+y^{2}}(F)$ and $K=K_{x^{2}+y^{2}}(F)$. Note that $(L, K) \in \Omega^{+}$because $\Delta(F)>0$ by (1.7). For $t \in \mathbb{R}$, a direct computation yields

$$
F_{T^{+}(t)}(x, y)=A(t) x^{4}+B(t) x^{3} y+C(t) x^{2} y^{2}-B(t) x y^{3}+A(t) y^{4},
$$

where

$$
\left\{\begin{array}{l}
A(t)=\frac{6 a_{4}+a_{2}}{8}+\frac{2 a_{4}-a_{2}}{8} \cos (4 t)-\frac{a_{3}}{4} \sin (4 t), \\
B(t)=a_{3} \cos (4 t)+\frac{2 a_{4}-a_{2}}{2} \sin (4 t), \\
C(t)=\frac{6 a_{4}+a_{2}}{4}-\frac{3\left(2 a_{4}-a_{2}\right)}{4} \cos (4 t)+\frac{3 a_{3}}{2} \sin (4 t) .
\end{array}\right.
$$

It is not hard to show that there exists a unique $t_{0} \in(-\pi / 4, \pi / 4]$ such that $B\left(t_{0}\right)=0$ and $2 A\left(t_{0}\right)-C\left(t_{0}\right)>0$. Put $(A, C)=\left(A\left(t_{0}\right), C\left(t_{0}\right)\right)$. Then, we have

$$
(L, K)=\left(L_{x^{2}+y^{2}}\left(F_{T^{+}\left(t_{0}\right)}\right), K_{x^{2}+y^{2}}\left(F_{T^{+}\left(t_{0}\right)}\right)\right)=(-6 A-C,-2 C(6 A-C))
$$

by Propositions 3.1 and $3.2(\mathrm{a})$. We solve that $F_{T^{+}\left(t_{0}\right)}=F_{(L, K)}$, or equivalently

$$
F=\left(F_{(L, K)}\right)_{T^{+}\left(-t_{0}\right)}=\Phi\left(L, K,-t_{0}\right) .
$$

Since $-t_{0} \in[-\pi / 4, \pi / 4)$ is uniquely determined by $F$, this shows that $\Phi$ is a bijection.

Finally, the above calculation also yields

$$
\left(\Theta_{1} \circ \Phi\right)(L, K, t)=\left(\Phi_{1}(L, K, t), \Phi_{2}(L, K, t), \Phi_{3}(L, K, t)\right),
$$

where

$$
\left\{\begin{array}{l}
\Phi_{1}(L, K, t)=-\frac{L}{8}+\frac{\sqrt{L^{2}+4 K}}{24} \cos (4 t), \\
\Phi_{2}(L, K, t)=\frac{\sqrt{L^{2}+4 K}}{6} \sin (4 t), \\
\Phi_{3}(L, K, t)=-\frac{L}{4}-\frac{\sqrt{L^{2}+4 K}}{4} \cos (4 t) .
\end{array}\right.
$$

By a direct computation, we then see that Proposition 4.2 (b) holds. 


\subsection{Indefinite case. Define}

$$
\left\{\begin{array}{lll}
\Phi^{(i)}: \Omega^{+} \times \mathbb{R} \longrightarrow V_{\mathbb{R}, x^{2}-y^{2}}^{0} ; & \Phi^{(i)}(L, K, t)=\left(F_{(L, K)}^{(i)}\right)_{T^{-}(t)} & \text { for } i=1,2, \\
\Phi^{(i)}: \Omega^{-} \times \mathbb{R} \longrightarrow V_{\mathbb{R}, x^{2}-y^{2}}^{0} ; & \Phi^{(i)}(L, K, t)=\left(F_{(L, K)}^{(i)}\right)_{T^{-}(t)} & \text { for } i=3,4,
\end{array}\right.
$$

where

$$
\begin{array}{r}
F_{(L, K)}^{(i)}(x, y)=\frac{3 L+(-1)^{i} \sqrt{L^{2}+4 K}}{24} x^{4}+\frac{-L+(-1)^{i} \sqrt{L^{2}+4 K}}{4} x^{2} y^{2} \\
+\frac{3 L+(-1)^{i} \sqrt{L^{2}+4 K}}{24} y^{4}
\end{array}
$$

for $i=1,2$, and

$$
F_{(L, K)}^{(i)}(x, y)=\frac{(-1)^{i} \sqrt{2 L^{2}-K}}{3} x^{3} y-L x^{2} y^{2}+\frac{(-1)^{i} \sqrt{2 L^{2}-K}}{3} x y^{3}
$$

for $i=3,4$. The images of $\Phi^{(1)}, \Phi^{(2)}, \Phi^{(3)}, \Phi^{(4)}$ lie in $V_{\mathbb{R}, x^{2}-y^{2}}$ by (3.1) and (1.8). Using Propositions 3.1 and 3.2 (a), it is easy to check that Proposition 4.3 (a) holds.

Now, by (3.1), an arbitrary $F \in V_{\mathbb{R}, x^{2}-y^{2}}^{0}$ has the shape

$$
F(x, y)=a_{4} x^{4}+a_{3} x^{3} y+a_{2} x^{2} y^{2}+a_{3} x y^{3}+a_{4} y^{4} .
$$

Write $L=L_{x^{2}-y^{2}}(F)$ and $K=K_{x^{2}-y^{2}}(F)$. For $t \in \mathbb{R}$, a direct computation yields

$$
F_{T^{-}(t)}(x, y)=A(t) x^{4}+B(t) x^{3} y+C(t) x^{2} y^{2}+B(t) x y^{3}+A(t) y^{4} \text {, }
$$

where

$$
\left\{\begin{array}{l}
A(t)=\frac{6 a_{4}-a_{2}}{8}+\frac{2 a_{4}+a_{2}}{8} \cosh (4 t)+\frac{a_{3}}{4} \sinh (4 t), \\
B(t)=a_{3} \cosh (4 t)+\frac{2 a_{4}+a_{2}}{2} \sinh (4 t), \\
C(t)=-\frac{6 a_{4}-a_{2}}{4}+\frac{3\left(2 a_{4}+a_{2}\right)}{4} \cosh (4 t)+\frac{3 a_{3}}{2} \sinh (4 t) .
\end{array}\right.
$$

Note that $\frac{d}{d t} A(t)=\frac{1}{2} B(t)$. It is not hard to check that:

- If $\Delta(F)>0$, then there is a unique $t_{0} \in \mathbb{R}$ such that $B\left(t_{0}\right)=0$.

- If $\Delta(F)<0$, then $B(t) \neq 0$ for all $t \in \mathbb{R}$, and there is a unique $t_{0} \in \mathbb{R}$ such that $A\left(t_{0}\right)=0$.

Put $(A, B, C)=\left(A\left(t_{0}\right), B\left(t_{0}\right), C\left(t_{0}\right)\right)$. Then, we have

$$
(L, K)=\left(L_{x^{2}-y^{2}}\left(F_{T^{-}\left(t_{0}\right)}\right), K_{x^{2}-y^{2}}\left(F_{T^{-}\left(t_{0}\right)}\right)\right)= \begin{cases}(6 A-C, 2 C(6 A+C)) & \text { if } \Delta(F)>0, \\ \left(-C,-9 B^{2}+2 C^{2}\right) & \text { if } \Delta(F)<0 .\end{cases}
$$

by Propositions 3.1 and $3.2(\mathrm{a})$. We solve that $F_{T^{-}\left(t_{0}\right)}=F_{(L, K)}^{(i)}$, or equivalently

$$
F=\left(F_{(L, K)}^{(i)}\right)_{T^{-}\left(-t_{0}\right)}=\Phi^{(i)}\left(L, K,-t_{0}\right) \text {, for exactly one } i \in\{1,2,3,4\} .
$$

Since $t_{0}$ is uniquely determined by $F$, this shows that $\Phi^{(1)}, \Phi^{(2)}, \Phi^{(3)}, \Phi^{(4)}$ are all injections, and that the stated disjoint union holds.

Finally, the above calculation also yields

$$
\left(\Theta_{1} \circ \Phi^{(i)}\right)(L, K, t)=\left(\Phi_{1}^{(i)}(L, K, t), \Phi_{2}^{(i)}(L, K, t), \Phi_{3}^{(i)}(L, K, t)\right),
$$


where

$$
\left\{\begin{array}{l}
\Phi_{1}^{(i)}(L, K, t)=\frac{L}{8}+\frac{(-1)^{i} \sqrt{L^{2}+4 K}}{24} \cosh (4 t), \\
\Phi_{2}^{(i)}(L, K, t)=\frac{(-1)^{i} \sqrt{L^{2}+4 K} \sinh (4 t),}{6} \\
\Phi_{3}^{(i)}(L, K, t)=-\frac{L}{4}+\frac{(-1)^{i} \sqrt{L^{2}+4 K}}{4} \cosh (4 t),
\end{array}\right.
$$

for $i=1,2$, and

$$
\left\{\begin{array}{l}
\Phi_{1}^{(i)}(L, K, t)=\frac{L}{8}-\frac{L}{8} \cosh (4 t)+\frac{(-1)^{i} \sqrt{2 L^{2}-K}}{12} \sinh (4 t), \\
\Phi_{2}^{(i)}(L, K, t)=\frac{(-1)^{i} \sqrt{2 L^{2}-K}}{3} \cosh (4 t)-\frac{L}{2} \sinh (4 t), \\
\Phi_{3}^{(i)}(L, K, t)=-\frac{L}{4}-\frac{3 L}{4} \cosh (4 t)+\frac{(-1)^{i} \sqrt{2 L^{2}-K}}{2} \sinh (4 t),
\end{array}\right.
$$

for $i=3,4$. By a direct computation, we then see that Proposition 4.3 (b) holds.

4.3. Reducible case. Suppose $\gamma=0$. For $t \in \mathbb{R}$, put

$$
T(t)=\left(\begin{array}{cc}
e^{-t} & 0 \\
\frac{2 \alpha \sinh t}{\beta} & e^{t}
\end{array}\right)
$$

which is an element of $O_{f}(\mathbb{R})$. Define

$$
\Phi_{f}^{(i)}: \Omega^{0} \times \mathbb{R} \longrightarrow V_{\mathbb{R}, f}^{0} ; \quad \Phi_{f}^{(i)}(L, K, t)=\left(F_{f,(L, K)}^{(i)}\right)_{T(t)} \quad \text { for } i=1,2,
$$

where

$$
\begin{array}{r}
F_{f,(L, K)}^{(i)}(x, y)=\left(\frac{L^{2}+(-1)^{i} 72 \alpha^{2} L+4 K+144 \alpha^{4}}{(-1)^{i} 144 \beta^{2}}\right) x^{4}+\left(\frac{\alpha L+(-1)^{i} 4 \alpha^{3}}{\beta}\right) x^{3} y \\
+\left(\frac{L+(-1)^{i} 12 \alpha^{2}}{2}\right) x^{2} y^{2}+(-1)^{i} 4 \alpha \beta x y^{3}+(-1)^{i} \beta^{2} y^{4} .
\end{array}
$$

The images of $\Phi_{f}^{(1)}, \Phi_{f}^{(2)}$ lie in $V_{\mathbb{R}, f}$ by (3.2) and (1.8). Using Propositions 3.1 and 3.2 (b), it is easy to check that Proposition 4.4 (a) holds.

Now, by (3.2), an arbitrary $F \in V_{\mathbb{R}, f}^{0}$ has the shape

$$
F(x, y)=a_{4} x^{4}+\left(\frac{2 \alpha\left(\beta^{2} a_{2}-4 \alpha^{2} a_{0}\right)}{\beta^{3}}\right) x^{3} y+a_{2} x^{2} y^{2}+\left(\frac{4 \alpha a_{0}}{\beta}\right) x y^{3}+a_{0} y^{4}
$$

Write $L=L_{f}(F)$ and $K=K_{f}(F)$. For $t \in \mathbb{R}$, a direct computation yields

$$
F_{T(t)}(x, y)=A(t) x^{4}+(*) x^{3} y+B(t) x^{2} y^{2}+(*) x y^{3}+C(t) y^{4},
$$


where

$$
\left\{\begin{array}{l}
A(t)=e^{-4 t} a_{4}+\frac{\alpha^{2}}{\beta^{2}}\left(e^{4 t}-1\right) e^{-4 t} a_{2}+\frac{\alpha^{4}}{\beta^{4}}\left(e^{4 t}-1\right)\left(e^{4 t}-5\right) e^{-4 t} a_{0}, \\
B(t)=a_{2}+\frac{6 \alpha^{2}}{\beta^{2}}\left(e^{4 t}-1\right) a_{0}, \\
C(t)=e^{4 t} a_{0} .
\end{array}\right.
$$

Since $\Delta(F) \neq 0$, we have $(-1)^{i} a_{0}>0$ for a unique $i \in\{1,2\}$, and there is a unique $t_{0} \in \mathbb{R}$ such that $C\left(t_{0}\right)=(-1)^{i} \beta^{2}$. Put $(A, B)=\left(A\left(t_{0}\right), B\left(t_{0}\right)\right)$. Then, we have

$$
\begin{aligned}
(L, K) & =\left(L_{f}\left(F_{T\left(t_{0}\right)}\right), K_{f}\left(F_{T\left(t_{0}\right)}\right)\right) \\
& =\left(2 B-(-1)^{i} 12 \alpha^{2},-B^{2}+(-1)^{i} 36 \beta^{2} A-(-1)^{i} 24 \alpha^{2} B+144 \alpha^{4}\right),
\end{aligned}
$$

by Propositions 3.1 and $3.2(\mathrm{~b})$. We solve that $F_{T\left(t_{0}\right)}=F_{f,(L, K)}^{(i)}$, or equivalently

$$
F=\left(F_{f,(L, K)}^{(i)}\right)_{T\left(-t_{0}\right)}=\Phi_{f}^{(i)}\left(L, K,-t_{0}\right) .
$$

Since $t_{0}$ and $i$ are uniquely determined by $F$, this shows that $\Phi_{f}^{(1)}$ and $\Phi_{f}^{(2)}$ are both injections, and that the stated disjoint union holds.

Finally, the above calculation also yields

$$
\left(\Theta_{2} \circ \Phi_{f}^{(i)}\right)(L, K, t)=\left(\Phi_{f, 1}^{(i)}(L, K, t), \Phi_{f, 2}^{(i)}(L, K, t), \Phi_{f, 3}^{(i)}(L, K, t)\right)
$$

where

$$
\left\{\begin{array}{l}
\Phi_{f, 1}^{(i)}(L, K, t)=\frac{(-1)^{i} e^{-4 t}}{144 \beta^{2}}\left(L^{2}+4 K\right)+\frac{\alpha^{2}}{2 \beta^{2}} L+\frac{(-1)^{i} \alpha^{4} e^{4 t}}{\beta^{2}} \\
\Phi_{f, 2}^{(i)}(L, K, t)=\frac{L}{2}+(-1)^{i} 6 \alpha^{2} e^{4 t} \\
\Phi_{f, 3}^{(i)}(L, K, t)=(-1)^{i} \beta^{2} e^{4 t} .
\end{array}\right.
$$

By a direct computation, we then see that Proposition 4.4 (b) holds.

\section{Definition of A BOUnded SEMI-AlgeBRAIC SET}

Throughout this section, let $f(x, y)=\alpha x^{2}+\beta x y+\gamma y^{2}$ be an integral and primitive binary quadratic form with $\Delta(f) \neq 0$ and $\alpha>0$, in the shape (1.11) whenever $f$ is reducible. As we have already explained in Subsection 1.1, the proof of Theorem 1.2 is reduced to counting points in the lattices in (1.10), which in turn amounts to certain volume computations, by the result below.

Proposition 5.1 (Davenport's lemma). Let $\mathcal{R}$ be a bounded semi-algebraic multi-set in $\mathbb{R}^{n}$ having maximum multiplicity $m$ and which is defined by at most $k$ polynomial inequalities, each having degree at most $\ell$. Then, the number of integral lattice points (counted with multiplicity) contained in the region $\mathcal{R}$ is

$$
\operatorname{Vol}(\mathcal{R})+O(\max \{\operatorname{Vol}(\overline{\mathcal{R}}), 1\})
$$

where $\operatorname{Vol}(\overline{\mathcal{R}})$ denotes the greatest d-dimensional volume of any projection of $\mathcal{R}$ onto a coordinate subspace by equating $n-d$ coordinates to zero, with $1 \leq d \leq n-1$. The implied constant in the second summand depends only on $n, m, k, \ell$. 
Proof. This is a result of Davenport [11], and the above formulation is due to Bhargava and Shankar in [4, Proposition 2.6].

For $X>0$, define

$$
V_{\mathbb{R}, f}^{0}(X)=\left\{F \in V_{\mathbb{R}, f}^{0}: H_{f}(F) \leq X\right\} \text { and } V_{\mathbb{Z}, f}^{0}(X)=\left\{F \in V_{\mathbb{Z}, f}^{0}: H_{f}(F) \leq X\right\} .
$$

However, to prove Theorem 1.2, we cannot apply Proposition 5.1 directly to

$$
\Theta_{w(f)}\left(V_{\mathbb{R}, f}^{0}(\mathbb{R})\right), \text { where } w(f)= \begin{cases}1 & \text { if } f \text { is irreducible, } \\ 2 & \text { if } f \text { is reducible, }\end{cases}
$$

as in Subsection 1.1, to count the lattice points in $\Theta_{w(f)}\left(V_{\mathbb{Z}, f}\right) \subset \Lambda_{f, w(f)}$ because

(1) the set $\Theta_{w(f)}\left(V_{\mathbb{R}, f}^{0}(X)\right)$ is unbounded when $f$ is indefinite,

(2) distinct forms in $V_{\mathbb{Z}, f}^{0}(X)$ might be $\mathrm{GL}_{2}(\mathbb{Z})$-equivalent.

Recall (4.1) and define

$$
\Omega^{*}(X)=\left\{(L, K) \in \Omega^{*}: \max \left\{L^{2},|K|\right\} \leq X\right\} \text { for } * \in\{0,+,-\} .
$$

In the notation of Lemma 4.1 as well as Propositions 4.2, 4.3, and 4.4, we have

$$
V_{\mathbb{R}, f}^{0}(X)=\left\{\begin{array}{l}
\left(\Psi_{f} \circ \Phi\right)\left(\Omega^{+}(X) \times[-\pi / 4, \pi / 4)\right), \\
\bigsqcup_{i=1}^{2}\left(\Psi_{f} \circ \Phi^{(i)}\right)\left(\Omega^{+}(X) \times \mathbb{R}\right) \sqcup \bigsqcup_{i=3}^{4}\left(\Psi_{f} \circ \Phi^{(i)}\right)\left(\Omega^{-}(X) \times \mathbb{R}\right), \\
\bigsqcup_{i=1}^{2} \Phi_{f}^{(i)}\left(\Omega^{0}(X) \times \mathbb{R}\right),
\end{array}\right.
$$

respectively, if $f$ is positive definite, indefinite, and reducible. We shall overcome the two issues above by restricting the values for $t \in \mathbb{R}$.

For brevity, in this section, write

$$
D_{f}=|\Delta(f)| \text { and } \delta_{f}=D_{f} / 4,
$$

as in Theorem 1.2 and Lemma 4.1, respectively.

Definition 5.2. If $f$ is positive definite, define

$$
\mathcal{S}_{f}(X)=\left(\Psi_{f} \circ \Phi\right)\left(\Omega^{+}(X) \times[-\pi / 4, \pi / 4)\right) .
$$

If $f$ is reducible, define

$$
\mathcal{S}_{f}(X)=\bigsqcup_{i=1}^{2} \Phi_{f}^{(i)}\left(\Omega^{0}(X) \times\left[t_{f, 1}, t_{f, 2}\right]\right) \text { for } t_{f, 1}=-\frac{\log 8}{4} \text { and } t_{f, 2}=\frac{\log (5 X / 18)}{4}
$$

If $f$ is indefinite and irreducible, define

$$
\mathcal{S}_{f}(X)=\bigsqcup_{i=1}^{2}\left(\Psi_{f} \circ \Phi^{(i)}\right)\left(\Omega^{+}(X) \times\left[0, t_{D_{f}}\right)\right) \sqcup \bigsqcup_{i=3}^{4}\left(\Psi_{f} \circ \Phi^{(i)}\right)\left(\Omega^{-}(X) \times\left[0, t_{D_{f}}\right)\right),
$$

where $t_{D_{f}}$ is defined as in Theorem 1.2 (c).

The goal of this section to prove the following preliminary results and estimates:

Proposition 5.3. The set $\Theta_{w(f)}\left(S_{f}(X)\right)$ is bounded, semi-algebraic, and definable by an absolutely bounded number of polynomial inequalities whose degrees are absolutely bounded. 
Proposition 5.4. The following statements hold.

(a) A form in $V_{\mathbb{Z}, f}^{0}(X)$ is $\mathrm{GL}_{2}(\mathbb{Z})$-equivalent to at least one form in $\mathcal{S}_{f}(X)$.

(b) A form in $V_{\mathbb{Z}, f}^{0}(X)$ for which $\Delta(F) \neq \square$ is $\mathrm{GL}_{2}(\mathbb{Z})$-equivalent to exactly $r_{f}$ forms in $\mathcal{S}_{f}(X)$, where $r_{f}$ is defined as in Theorem 1.2.

5.1. Alternative description. First, we shall give an alternative description of the set $\mathcal{S}_{f}(X)$ in terms of the coefficients of the forms in $V_{\mathbb{R}, f}^{0}(X)$.

Lemma 5.5. If $f$ is positive definite, then $\mathcal{S}_{f}(X)=V_{\mathbb{R}, f}^{0}(X)$.

Proof. This is clear from (5.1).

Lemma 5.6. If $f$ is reducible, then

$$
\mathcal{S}_{f}(X)=\left\{F \in V_{\mathbb{R}, f}^{0}(X): \beta^{2} / 8 \leq\left|C_{F}\right| \leq 5 \beta^{2} X / 18\right\},
$$

where $C_{F}$ denotes the $y^{4}$-coefficient of $F$.

Proof. For $i=1,2$ and for any $F=\Phi_{f}^{(i)}(L, K, t)$, we have $C_{F}=(-1)^{i} \beta^{2} e^{4 t}$ by (4.11), and the claim is then clear from (5.1).

Lemma 5.7. If $f$ is an indefinite and irreducible, then

$$
\mathcal{S}_{f}(X)=\left\{F \in V_{\mathbb{R}, f}^{0}(X): 1 \leq E_{f, 1}(F) Z_{f}(F) / E_{f, 2}(F)<e^{8 t_{D_{f}}}\right\},
$$

where in the notation of Proposition 3.2 (a), we define

$$
E_{f, 1}(F)=L_{f, 1}(F)-\sqrt{D_{f}} L_{f, 2}(F) \text { and } E_{f, 2}(F)=L_{f, 1}(F)+\sqrt{D_{f}} L_{f, 2}(F),
$$

and for $F$ in the image of $\Psi_{f} \circ \Phi^{(i)}$, we define

$$
Z_{f}(F)= \begin{cases}1 & \text { for } i=1,2, \\ \frac{L_{f}(F)^{2}+4 K_{f}(F)}{\left(4 L_{f}(F)-(-1)^{i} 2 \sqrt{2 L_{f}(F)^{2}-K_{f}(F)}\right)^{2}} & \text { for } i=3,4 .\end{cases}
$$

Proof. For $i=1,2,3,4$, consider $F=\left(\Psi_{f} \circ \Phi^{(i)}\right)(L, K, t)$. For $k=1,2$, we have

$$
E_{f, k}(F)= \begin{cases}(-1)^{i} 2 \alpha^{2} \sqrt{L_{f}(F)^{2}+4 K_{f}(F)} e^{(-1)^{k+1} 4 t} / 3 & \text { if } i=1,2, \\ -2 \alpha^{2}\left(3 L_{f}(F)+(-1)^{k+i} 2 \sqrt{2 L_{f}(F)^{2}-K_{f}(F)}\right) e^{(-1)^{k+1} 4 t} / 3 & \text { if } i=3,4\end{cases}
$$

by a direct computation using (4.2), (4.7), and (4.8). We then see that

$$
E_{f, 1}(F) Z_{f}(F) / E_{f, 2}(F)=e^{8 t}
$$

from which the claim follows.

5.2. Proof of Proposition 5.3. From (4.5), (4.7), (4.8), and (4.11), it is clear that the set $\mathcal{S}_{f}(X)$ is bounded. Thus, it remains to show that $\mathcal{S}_{f}(X)$ is a semi-algebraic set definable by an absolutely bounded number of polynomial inequalities whose degrees are absolutely bounded.

5.2.1. The case when $f$ is positive definite or reducible. The claim follows immediately from Lemmas 5.5 and 5.6 as well as Proposition 3.2. 
5.2.2. The case when $f$ is indefinite and irreducible. The only problem is that $Z_{f}(F)$ is not a polynomial in the $x^{4}, x^{3} y$, and $x^{2} y^{2}$-coefficients of $F$. We shall resolve this issue in Lemma 5.8 below. The claim then follows from Lemma 5.7 and Proposition 3.2.

Lemma 5.8. For $i=3,4$, let $F \in\left(\Psi_{f} \circ \Phi^{(i)}\right)\left(\Omega^{-} \times \mathbb{R}\right)$. Then, the condition

$$
1 \leq E_{f, 1}(F) Z_{f}(F) / E_{f, 2}(F)<e^{8 t_{D_{f}}}
$$

is equivalent to an absolutely bounded number of polynomial inequalities in the variables $L_{f}(F), K_{f}(F), E_{f, 1}(F), E_{f, 2}(F)$ whose degrees are absolutely bounded.

Proof. For brevity, define

$$
\begin{aligned}
& Y_{f, 1}(F)=-E_{f, 1}(F)\left(L_{f}(F)^{2}+4 K(F)\right)+E_{f, 2}(F)\left(17 L_{f}(F)^{2}-4 K_{f}(F)\right), \\
& Y_{f, 2}(F)=-E_{f, 1}(F)\left(L_{f}(F)^{2}+4 K_{f}(F)\right)+e^{8 t_{D_{f}}} E_{f, 2}(F)\left(17 L_{f}(F)^{2}-4 K_{f}(F)\right),
\end{aligned}
$$

as well as write

$$
\left(L, K, E_{1}, E_{2}, Z, Y_{1}, Y_{2}\right)=\left(L_{f}(F), K_{f}(F), E_{f, 1}(F), E_{f, 2}(F), Z_{f}(F), Y_{f, 1}(F), Y_{f, 2}(F)\right) .
$$

Note that $L^{2}+4 K<0$ by $(1.7)$ because $\Delta(F)<0$. This implies that $Z<0$ and so the stated condition may be rewritten as

$$
\begin{cases}E_{2} \leq E_{1} Z<e^{8 t_{D_{f}}} E_{2} & \text { if } E_{2}>0, \text { which is equivalent to } i=3, \\ E_{2} \geq E_{1} Z>e^{8 t_{D_{f}}} E_{2} & \text { if } E_{2}<0, \text { which is equivalent to } i=4 .\end{cases}
$$

By rearranging, we may further rewrite the above as

$$
12 E_{2} L \sqrt{2 L^{2}-K} \leq(-1)^{i} Y_{1} \text { and } 12 e^{8 t_{D_{f}}} E_{2} L \sqrt{2 L^{2}-K}>(-1)^{i} Y_{2} .
$$

From here, we shall consider the different possibilities for the signs of $E_{2}, L, Y_{1}, Y_{2}$. For example, when $E_{2}>0$ and $L \geq 0$, the above is equivalent to $Y_{1} \leq 0$ and

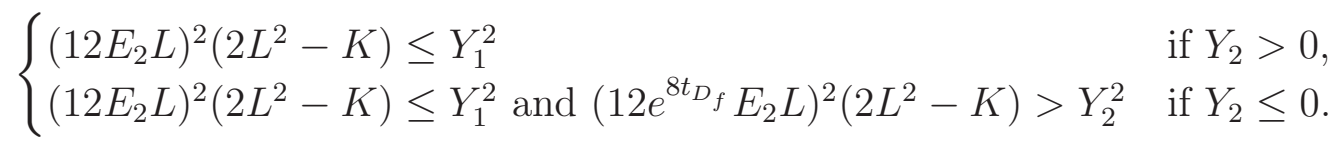

The other cases are analogous. We then see that the claim holds.

5.3. Integral orthogonal groups. We shall require an explicit description of

$$
O_{f}(\mathbb{Z})=O_{f}(\mathbb{R}) \cap \mathrm{GL}_{2}(\mathbb{Z}) .
$$

In the notation of Lemma 4.1, observe that

$$
O_{f}(\mathbb{R})= \begin{cases}T_{f}^{-1}\left(O_{x^{2}+y^{2}}(\mathbb{R})\right) T_{f} & \text { if } f \text { is positive definite, } \\ T_{f}^{-1}\left(O_{x^{2}-y^{2}}(\mathbb{R})\right) T_{f} & \text { if } f \text { is indefinite. }\end{cases}
$$

Moreover, it is well-known that

$$
\begin{aligned}
& O_{x^{2}+y^{2}}(\mathbb{R})=\left\{J_{k} T^{+}(t): k \in\{1,4\} \text { and } t \in \mathbb{R}\right\}, \\
& O_{x^{2}-y^{2}}(\mathbb{R})=\left\{ \pm J_{k} T^{-}(t): k \in\{1,2,3,4\} \text { and } t \in \mathbb{R}\right\},
\end{aligned}
$$

where $T^{+}(t)$ and $T^{-}(t)$ are defined as in (4.3), and

$$
J_{1}=\left(\begin{array}{ll}
1 & 0 \\
0 & 1
\end{array}\right), J_{2}=\left(\begin{array}{ll}
0 & 1 \\
1 & 0
\end{array}\right), J_{3}=\left(\begin{array}{cc}
0 & 1 \\
-1 & 0
\end{array}\right), J_{4}=\left(\begin{array}{cc}
1 & 0 \\
0 & -1
\end{array}\right) .
$$

We shall need the following lemma. 
Lemma 5.9. Suppose that $T \in O_{f}(\mathbb{Z}) \backslash\left\{ \pm I_{2 \times 2}\right\}$ has finite order. Then, the form $f$ is $\mathrm{GL}_{2}(\mathbb{Z})$-equivalent to a form of the shape

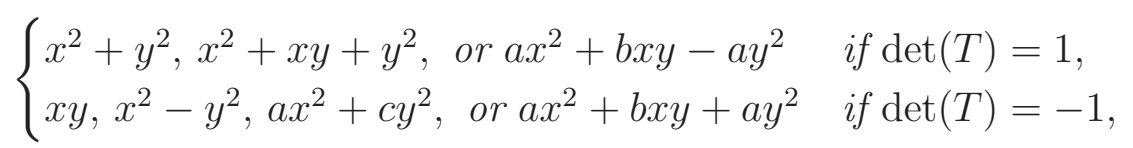

for some integers $a, b$, and $c$.

Proof. By [21, Chapter IX], for example, a finite cyclic subgroup of $\mathrm{GL}_{2}(\mathbb{Z})$ not contained in $\left\{ \pm I_{2 \times 2}\right\}$ is conjugate to the subgroup generated by one of the following:

$$
\left(\begin{array}{cc}
0 & 1 \\
-1 & -1
\end{array}\right),\left(\begin{array}{cc}
0 & 1 \\
-1 & 0
\end{array}\right),\left(\begin{array}{cc}
0 & -1 \\
1 & 1
\end{array}\right),\left(\begin{array}{cc}
1 & 0 \\
0 & -1
\end{array}\right),\left(\begin{array}{ll}
0 & 1 \\
1 & 0
\end{array}\right) \text {. }
$$

We then deduce that there exists $P \in \mathrm{GL}_{2}(\mathbb{Z})$ such that $Q=P^{-1} T P$ is equal to one of the following matrices up to sign:

$$
\left(\begin{array}{cc}
0 & 1 \\
-1 & -1
\end{array}\right),\left(\begin{array}{cc}
-1 & -1 \\
1 & 0
\end{array}\right),\left(\begin{array}{cc}
0 & 1 \\
-1 & 0
\end{array}\right),\left(\begin{array}{cc}
1 & 0 \\
0 & -1
\end{array}\right),\left(\begin{array}{ll}
0 & 1 \\
1 & 0
\end{array}\right)
$$

Since $f$ is primitive with $\alpha>0$ by assumption and $\left(f_{P}\right)_{Q}= \pm f_{P}$, we then check that $f_{P}$ must have one of the stated shapes.

Proposition 5.10. Suppose that $f$ is positive definite. Then, we have

$$
O_{f}(\mathbb{Z})=\left\{ \pm I_{2 \times 2}\right\}
$$

if $f$ is not $\mathrm{GL}_{2}(\mathbb{Z})$-equivalent to the forms below, and the group $O_{f}(\mathbb{Z})$ is equal to

$$
\left\{\begin{array}{cl}
\left\{ \pm I_{2 \times 2}, \pm\left(\begin{array}{cc}
0 & 1 \\
-1 & 0
\end{array}\right), \pm\left(\begin{array}{cc}
1 & 0 \\
0 & -1
\end{array}\right), \pm\left(\begin{array}{ll}
0 & 1 \\
1 & 0
\end{array}\right)\right\} & \text { if } f(x, y)=x^{2}+y^{2} \\
\left\{ \pm I_{2 \times 2} \pm\left(\begin{array}{cc}
1 & 1 \\
-1 & 0
\end{array}\right), \pm\left(\begin{array}{cc}
0 & -1 \\
1 & 1
\end{array}\right),\right. & \text { if } f(x, y)=x^{2}+x y+y^{2} \\
\left. \pm\left(\begin{array}{cc}
1 & 1 \\
0 & -1
\end{array}\right), \pm\left(\begin{array}{cc}
0 & 1 \\
1 & 0
\end{array}\right), \pm\left(\begin{array}{cc}
-1 & 0 \\
1 & 1
\end{array}\right)\right\} & \\
\left\{ \pm I_{2 \times 2}, \pm\left(\begin{array}{cc}
1 & 0 \\
0 & -1
\end{array}\right)\right\} & \text { if } f(x, y)=\alpha x^{2}+\gamma y^{2} \text { for } \alpha \neq \gamma, \\
\left\{ \pm I_{2 \times 2}, \pm\left(\begin{array}{ll}
0 & 1 \\
1 & 0
\end{array}\right)\right\} & \text { if } f(x, y)=\alpha x^{2}+\beta x y+\alpha y^{2} \text { for } \beta \notin\{0, \alpha\} .
\end{array}\right.
$$

Proof. Elements in $O_{f}(\mathbb{Z})$ have finite order by (5.2) and so the first claim follows from Lemma 5.9. Using (5.2), we compute that elements in $O_{f}(\mathbb{R})$ are of the forms

$$
\left(\begin{array}{cc}
\phi_{t}+\frac{\beta \psi_{t}}{2 \sqrt{\delta_{f}}} & \frac{\gamma \psi_{t}}{\sqrt{\delta_{f}}} \\
-\frac{\alpha \psi_{t}}{\sqrt{\delta_{f}}} & \phi_{t}-\frac{\beta \psi_{t}}{2 \sqrt{\delta_{f}}}
\end{array}\right) \text { and }\left(\begin{array}{cc}
\phi_{t}-\frac{\beta \psi_{t}}{2 \sqrt{\delta_{f}}} & \frac{\beta}{\alpha}\left(\phi_{t}-\frac{\beta \psi_{t}}{2 \sqrt{\delta_{f}}}\right)+\frac{\gamma \psi_{t}}{\sqrt{\delta_{f}}} \\
\frac{\alpha \psi_{t}}{\sqrt{\delta_{f}}} & -\phi_{t}-\frac{\beta \psi_{t}}{2 \sqrt{\delta_{f}}}
\end{array}\right)
$$

where $t \in \mathbb{R}$ and $\left(\phi_{t}, \psi_{t}\right)=(\cos t, \sin t)$. With the help of the proof of Lemma 5.9, it is not hard to check that $O_{f}(\mathbb{Z})$ is as claimed.

Proposition 5.11. Suppose that $f$ is reducible. Then, the group $O_{f}(\mathbb{Z})$ is equal to

$$
\begin{cases}\left\{ \pm I_{2 \times 2}\right\} & \text { if } \beta \nmid \alpha^{2}+1 \text { and } \beta \nmid \alpha^{2}-1, \\
\left\{ \pm I_{2 \times 2}, \pm\left(\begin{array}{cc}
\alpha & \beta \\
-\frac{\alpha^{2}+1}{\beta} & -\alpha
\end{array}\right)\right\} & \text { if } \beta \mid \alpha^{2}+1 \text { and } \beta \nmid \alpha^{2}-1, \\
\left\{ \pm I_{2 \times 2}, \pm\left(\begin{array}{cc}
\alpha & \beta \\
-\frac{\alpha^{2}-1}{\beta} & -\alpha
\end{array}\right)\right\} & \text { if } \beta \nmid \alpha^{2}+1 \text { and } \beta \mid \alpha^{2}-1, \\
\left\{ \pm I_{2 \times 2}, \pm\left(\begin{array}{cc}
-1 & 0 \\
2 & 1
\end{array}\right), \pm\left(\begin{array}{cc}
1 & 1 \\
-2 & -1
\end{array}\right), \pm\left(\begin{array}{cc}
1 & 1 \\
0 & -1
\end{array}\right)\right\} & \text { if } f(x, y)=x^{2}+x y \\
\left\{ \pm I_{2 \times 2}, \pm\left(\begin{array}{cc}
-1 & 0 \\
1 & 1
\end{array}\right), \pm\left(\begin{array}{cc}
1 & 2 \\
-1 & -1
\end{array}\right),\left(\begin{array}{cc}
1 & 2 \\
0 & -1
\end{array}\right)\right\} & \text { if } f(x, y)=x^{2}+2 x y .\end{cases}
$$


Proof. Using (5.2), we compute that elements in $O_{f}(\mathbb{R})$ are of the forms

$$
\pm\left(\begin{array}{cc}
\phi_{t}-\psi_{t} & 0 \\
\frac{2 \alpha \psi_{t}}{\beta} & \phi_{t}+\psi_{t}
\end{array}\right) \text { and } \pm\left(\begin{array}{cc}
\phi_{t}+\psi_{t} & \frac{\beta}{\alpha}\left(\phi_{t}+\psi_{t}\right) \\
-\frac{2 \alpha \psi_{t}}{\beta} & -\phi_{t}-\psi_{t}
\end{array}\right)
$$

where $t \in \mathbb{R}$ and $\left(\phi_{t}, \psi_{t}\right) \in\{(\cosh t, \sinh t),(\sinh t, \cosh t)\}$. For the matrix on the left to have integer entries, necessarily

$$
2 \cosh t, 2 \sinh t \in \mathbb{Z} \text { so }(2 \cosh t, 2 \sinh t)=(2,0) .
$$

Similarly, for the matrix on the right to have integer entries, necessarily

$2 \alpha \cosh t, 2 \alpha \sinh t,(\cosh t+\sinh t) / \alpha \in \mathbb{Z}$ so $(2 \alpha \cosh t, 2 \alpha \sinh t)=\left(\alpha^{2}+1, \alpha^{2}-1\right)$.

We then deduce that

$$
O_{f}(\mathbb{Z})=\left\{ \pm I_{2 \times 2}, \pm\left(\begin{array}{cc}
-1 & 0 \\
2 \alpha / \beta & 1
\end{array}\right),\left(\begin{array}{cc}
\alpha & \beta \\
-\left(\alpha^{2} \pm 1\right) / \beta & -\alpha
\end{array}\right)\right\} \cap \mathrm{GL}_{2}(\mathbb{Z})
$$

Since $f$ has the shape (1.11) by assumption, we have

$$
\beta \mid \alpha^{2}+1 \text { and } \beta \mid \alpha^{2}-1 \Longleftrightarrow \alpha=1 \text { and } \beta \in\{1,2\} \text {, }
$$

and we see that the claim indeed holds.

Proposition 5.12. Suppose that $f$ is indefinite and irreducible. Define

$$
G_{f}(\mathbb{Z})=\left\{ \pm T_{D_{f}}^{n}: n \in \mathbb{Z}\right\} \text {, where } T_{D_{f}}=\left(\begin{array}{cc}
\frac{u_{D_{f}}-\beta v_{D_{f}}}{2} & -\gamma v_{D_{f}} \\
\alpha v_{D_{f}} & \frac{u_{D_{f}}+\beta v_{D_{f}}}{2}
\end{array}\right)
$$

and $\left(u_{D_{f}}, v_{D_{f}}\right) \in \mathbb{N}^{2}$ is the least solution to $x^{2}-D_{f} y^{2}= \pm 4$. Then, we have

$$
O_{f}(\mathbb{Z})=G_{f}(\mathbb{Z})
$$

if $f$ is not $\mathrm{GL}_{2}(\mathbb{Z})$-equivalence to the forms below, and the group $O_{f}(\mathbb{Z})$ is equal to

$$
\begin{cases}G_{f}(\mathbb{Z}) \sqcup G_{f}(\mathbb{Z})\left(\begin{array}{cc}
1 & \beta / \alpha \\
0 & -1
\end{array}\right) & \text { if } f(x, y)=\alpha x^{2}+\beta x y+\gamma y^{2} \text { with } \alpha \mid \beta, \\
G_{f}(\mathbb{Z}) \sqcup G_{f}(\mathbb{Z})\left(\begin{array}{cc}
0 & 1 \\
-1 & 0
\end{array}\right) & \text { if } f(x, y)=\alpha x^{2}+\beta x y-\alpha y^{2} .\end{cases}
$$

Proof. By (5.2), elements in $O_{f}(\mathbb{R})$ of infinite order are of the shape

$$
\pm\left(\begin{array}{cc}
\phi_{t}-\frac{\beta \psi_{t}}{2 \sqrt{\delta_{f}}} & -\frac{\gamma \psi_{t}}{\sqrt{\delta_{f}}} \\
\frac{\alpha \psi_{t}}{\sqrt{\delta_{f}}} & \phi_{t}+\frac{\beta \psi_{t}}{2 \sqrt{\delta_{f}}}
\end{array}\right)
$$

where $t \in \mathbb{R}$ and $\left(\phi_{t}, \psi_{t}\right) \in\{(\cosh t, \sinh t),(\sinh t, \cosh t)\}$. We then see that

$$
G_{f}(\mathbb{Z})=\left\{ \pm I_{2 \times 2}\right\} \sqcup\left\{T \in O_{f}(\mathbb{Z}): T \text { has infinite order }\right\} .
$$

Hence, the first claim follows from Lemma 5.9 and the fact that $a x^{2}+b x y+a y^{2}$ is $\mathrm{GL}_{2}(\mathbb{Z})$-equivalent to the form

$$
(2 a-b) x^{2}+(2 a-b) x y+a y^{2} \text { via }\left(\begin{array}{cc}
-1 & -1 \\
1 & 0
\end{array}\right) .
$$

Now, again by $(5.2)$, elements in $O_{f}(\mathbb{R})$ of finite order have the shape

$$
\left(\begin{array}{cc}
\frac{-\beta}{\sqrt{D_{f}}} & -\frac{2 \gamma}{\sqrt{D_{f}}} \\
\frac{2 \alpha}{\sqrt{D_{f}}} & \frac{\beta}{\sqrt{D_{f}}}
\end{array}\right) \text { and }\left(\begin{array}{cc}
\phi_{t}+\frac{\beta \psi_{t}}{2 \sqrt{\delta_{f}}} & \frac{\beta}{\alpha}\left(\phi_{t}+\frac{\beta \psi_{t}}{2 \sqrt{\delta_{f}}}\right)-\frac{\gamma \psi_{t}}{\sqrt{\delta_{f}}} \\
-\frac{\alpha \psi_{t}}{\sqrt{\delta_{f}}} & -\phi_{t}-\frac{\beta \psi_{t}}{2 \sqrt{\delta_{f}}},
\end{array}\right)
$$


where $t \in \mathbb{R}$ and $\left(\phi_{t}, \psi_{t}\right) \in\{(\cosh t, \sinh t),(\sinh t, \cosh t)\}$. Notice that the matrix on the left cannot lie in $\mathrm{GL}_{2}(\mathbb{Z})$ because $D_{f}$ is not square when $f$ is irreducible. Using the description of $O_{x^{2}-y^{2}}(\mathbb{R})$, it is then not hard to check that $\left[O_{f}(\mathbb{Z}): G_{f}(\mathbb{Z})\right] \leq 2$, from which the second claim follows.

5.4. Proof of Theorem 1.4. Suppose that $f(x, y)=\alpha x^{2}+\beta x y-\alpha y^{2}$ and that $D_{f}$ is not a square. In the notation of Proposition 5.12, we have

$$
x^{2}-D_{f} y^{2}=-4 \text { has integer solutions if and only if } \operatorname{det}\left(T_{D_{f}}\right)=-1
$$

by definition. But Proposition 5.12 also implies that $\operatorname{det}\left(T_{D_{f}}\right)=-1$ is equivalent to

$$
O_{f}(\mathbb{Z}) \text { has an element of finite order and negative determinant. }
$$

The theorem now follows from Lemma 5.9 and (5.4).

5.5. Proof of Proposition 5.4. We shall need the following lemma.

Lemma 5.13. For all $F \in V_{\mathbb{Z}, f}^{0}$ with $\Delta(F) \neq \square$ and $T \in \mathrm{GL}_{2}(\mathbb{Z}) \backslash\left\{ \pm I_{2 \times 2}\right\}$, we have

(a) $F_{T} \in V_{\mathbb{Z}, f}^{0}$ if and only if $T \in O_{f}(\mathbb{Z})$,

(b) $F_{T}=F$ if and only if $T= \pm D_{f}^{-1 / 2} M_{f}$.

Proof. Note that $F_{T} \in V_{\mathbb{Z}, f_{T}}^{0}$ by (1.8). By Theorem 1.1 (a), we then have $F_{T} \in V_{\mathbb{Z}, f}^{0}$ if and only if $f_{T}= \pm f$, whence part (a) holds. By Theorem 1.1 (a) and Proposition 2.1, we have $F_{T}=F$ if and only if $T$ is proportional to $M_{f}$, from which part (b) follows since $\operatorname{det}(T)= \pm 1$.

5.5.1. The case when $f$ is positive definite or reducible. Let us first observe that:

Lemma 5.14. We have $V_{\mathbb{Z}, f}^{0}(X) \subset \mathcal{S}_{f}(X)$.

Proof. Let $F \in V_{\mathbb{Z}, f}^{0}(X)$ be given. If $f$ is positive definite, then clearly $F \in \mathcal{S}_{f}(X)$ by Lemma 5.5. If $f$ is reducible, then recall Lemma 5.6, and we have $F \in \mathcal{S}_{f}(X)$ since

$$
\frac{8 C_{F}}{\beta^{2}} \in \mathbb{Z} \text { and }\left|\frac{8 C_{F}}{\beta^{2}}\right| \leq\left|\frac{4\left(L_{f}(F)^{2}+4 K_{f}(F)\right)}{9}\right| \leq \frac{20 X}{9}
$$

by (4.10) and Proposition 3.2 (b), respectively.

Lemma 5.14 implies that part (a) holds. Together with Lemma 5.13 (a, it further implies that for $F \in V_{\mathbb{Z}, f}^{0}(X)$ with $\Delta(F) \neq \square$, the number of forms in $\mathcal{S}_{f}(X)$ which are $\mathrm{GL}_{2}(\mathbb{Z})$-equivalent to $F$ is equal to

$$
\left[O_{f}(\mathbb{Z}): \operatorname{Stab}_{O_{f}(\mathbb{Z})}(F)\right]
$$

By Lemma 5.13 (b), we in turn have

$$
\left[O_{f}(\mathbb{Z}): \operatorname{Stab}_{O_{f}(\mathbb{Z})}(F)\right]=\left[O_{f}(\mathbb{Z}): O_{f}(\mathbb{Z}) \cap\left\{ \pm I_{2 \times 2}, \pm D_{f}^{-1 / 2} M_{f}\right\}\right],
$$

which may be verified to be equal to $r_{f}$ using Propositions 5.10 and 5.11. 
5.5.2. The case when $f$ is indefinite and irreducible. We shall use the notation from Lemma 4.1, Proposition 5.12, (4.3), and (5.3). Then, by definition, we have

$$
T_{D_{f}}=T_{f}^{-1} J_{k(f)} T^{-}\left(t_{D_{f}}\right) T_{f}, \text { where } k(f)= \begin{cases}1 & \text { if } u_{D_{f}}^{2}-D_{f} v_{D_{f}}^{2}=-4, \\ 2 & \text { if } u_{D_{f}}^{2}-D_{f} v_{D_{f}}^{2}=4,\end{cases}
$$

Now, by (5.1) and (4.6), a form in $V_{\mathbb{Z}, f}^{0}(X)$ is of the shape

$$
F=\left(F_{(L, K)}^{(i)}\right)_{T^{-}(t) T_{f}}, \text { where }(L, K, t) \in \Omega^{0}(X) \times \mathbb{R} \text { and } i \in\{1,2,3,4\} .
$$

Observe that $J_{1}$ and $J_{2}$ commute with $T^{-}(t)$ as well as fix the forms in $V_{\mathbb{R}, x^{2}-y^{2}}$. For any $n \in \mathbb{Z}$, we then deduce that

$$
F_{T_{D_{f}}^{n}}=\left(F_{(L, K)}^{(i)}\right)_{T^{-}(t) J_{k(f)}^{n} T^{-}\left(n t_{D_{f}}\right) T_{f}}=\left(F_{(L, K)}^{(i)}\right)_{T^{-}\left(t+n t_{D_{f}}\right) T_{f}} .
$$

Let $n_{1} \in \mathbb{Z}$ be the unique integer such that $0 \leq t+n_{1} t_{D_{f}}<t_{D_{f}}$. The existence of $n_{1}$ then implies part (a).

Next, suppose that $\Delta(F) \neq \square$, in which case

$$
\text { for } T \in \mathrm{GL}_{2}(\mathbb{Z}): F_{T} \in V_{\mathbb{Z}, f}^{0} \text { if and only if } T \in O_{f}(\mathbb{Z})
$$

by Lemma 5.13 (a). If $O_{f}(\mathbb{Z})=G_{f}(\mathbb{Z})$, then part (b) holds by the uniqueness of $n_{1}$. If $O_{f}(\mathbb{Z}) \neq G_{f}(\mathbb{Z})$, then recall from Proposition 5.12 that

$$
O_{f}(\mathbb{Z})=G_{f}(\mathbb{Z}) \sqcup G_{f}(\mathbb{Z}) M \text {, where } M \text { has finite order. }
$$

From (5.2), we see that

$$
M= \pm T_{f}^{-1} J_{k_{0}} T^{-}\left(t_{0}\right) T_{f}, \text { where } t_{0} \in \mathbb{R} \text { and } k_{0} \in\{3,4\} .
$$

Then, for any $n \in \mathbb{Z}$, it is straightforward to verify that

$$
\begin{aligned}
F_{T_{D_{f}}^{n} M}= & \left(F_{(L, K)}^{(i)}\right)_{T^{-}\left(t+n t_{D_{f}}\right) J_{k_{0}} T^{-}\left(t_{0}\right) T_{f}} \\
= & \begin{cases}\left(F_{(L, K)}^{(i)}\right)_{T^{-}\left(-\left(t+n t_{D_{f}}\right)+t_{0}\right) T_{f}} & \text { for } i \in\{1,2\}, \\
\left(F_{(L, K)}^{(j)}\right)_{T^{-}\left(-\left(t+n t_{D_{f}}\right)+t_{0}\right) T_{f}} & \text { for } i \in\{3,4\}, \text { where } j \in\{3,4\} \backslash\{i\} .\end{cases}
\end{aligned}
$$

There is a unique $n_{2} \in \mathbb{Z}$ such that $0 \leq-\left(t+n_{2} t_{D_{f}}\right)+t_{0}<t_{D_{f}}$. Observe that

$$
F_{T_{D_{f}}^{n_{1}}}=F_{T_{D_{f}}^{n_{2}} M} \text { would imply } F_{T_{D_{f}}^{n_{1}}}=\left(F_{T_{D_{f}}^{n_{1}}}\right)_{T_{D_{f}}^{n_{2}-n_{1}} M} \cdot
$$

But $T_{D_{f}}^{n_{2}-n_{1}} M$ has finite order, and so it cannot proportional to $M_{f}$ by (5.5), which is a contradiction by Lemma 5.13 (b). Then, we conclude from Proposition 5.12 that part (b) indeed holds.

\section{ERror estimates AND the MAin theOrem}

Throughout this section, let $f(x, y)=\alpha x^{2}+\beta x y+\gamma y^{2}$ be an integral and primitive binary quadratic form with $\Delta(f) \neq 0$ and $\alpha>0$, in the shape (1.11) whenever $f$ is reducible. Let $D_{f}, r_{f}$ and $s_{f}$ be as in Theorem 1.2.

In Subsections 6.1 and 6.2, respectively, we shall first prove: 
Proposition 6.1. For any $\epsilon>0$, we have

$$
\#\left\{F \in \mathcal{S}_{f}(X) \cap V_{\mathbb{Z}, f}^{0}: L_{f}(F)^{2}+4 K_{f}(F)=\square\right\}=O_{f, \epsilon}\left(X^{1+\epsilon}\right),
$$

and

$$
\begin{array}{r}
\#\left\{F \in \mathcal{S}_{f}(X) \cap V_{\mathbb{Z}, f}^{0}:\left(L_{f}(F)^{2}+4 K_{f}(F)\right)\left(2 L_{f}(F)-K_{f}(F)\right) / \Delta(f)=\square\right. \\
\text { and } \left.L_{f}(F) \neq 0\right\}=O_{f}\left(X^{1 / 2+\epsilon}\right) .
\end{array}
$$

Further, the number

$$
\#\left\{F \in \mathcal{S}_{f}(X) \cap V_{\mathbb{Z}, f}^{0}:-4 K_{f}(F) / \Delta(f)=\square \text { and } L_{f}(F)=0\right\}
$$

is equal to zero if $-\Delta(f) \neq \square$. and is bounded by $O_{f}(X)$ otherwise.

Propositions 6.1, 3.6, and 5.4 then imply part d) of Theorem 1.2.

The reader should compare the last claim above with [28, Theorem 1.4].

Proposition 6.2. We have

$$
\#\left\{F \in \mathcal{S}_{f}(X) \cap V_{\mathbb{Z}, f}^{0}: F \text { is reducible }\right\}= \begin{cases}O_{f}\left(X(\log X)^{2}\right) & \text { if } f \text { is irreducible } \\ O_{f}\left(X(\log X)^{3}\right) & \text { if } f \text { is reducible. }\end{cases}
$$

Now, from Propositions 5.4, 6.1, and 6.2, we also easily see that

$$
N_{\mathbb{Z}, f}^{\left(D_{4}\right)}(X)=\frac{1}{r_{f}} \#\left(S_{f}(X) \cap V_{\mathbb{Z}, f}^{0}\right)+O_{f, \epsilon}\left(X^{1+\epsilon}\right) \text { for any } \epsilon>0 .
$$

Let $\mathcal{L}_{f, w(f)}$ be a linear transformation on $\mathbb{R}^{3}$ which takes $\Lambda_{f, w(f)}$ to $\mathbb{Z}^{3}$, and define

$$
\mathcal{R}_{f}(X)=\left(\mathcal{L}_{f, w(f)} \circ \Theta_{w(f)}\right)\left(\mathcal{S}_{f}(X)\right), \text { where } w(f)= \begin{cases}1 & \text { if } f \text { is irreducible } \\ 2 & \text { if } f \text { is reducible }\end{cases}
$$

as before. Observe that then

$$
\#\left(\mathcal{S}_{f}(X) \cap V_{\mathbb{Z}, f}^{0}\right)=\#\left(\Theta_{w(f)}\left(\mathcal{S}_{f}(X)\right) \cap \Lambda_{f, w(f)}\right)=\#\left(\mathcal{R}_{f}(X) \cap \mathbb{Z}^{3}\right) .
$$

By Proposition 5.3, we may apply Proposition 5.1 to obtain

$$
\begin{aligned}
\#\left(S_{f}(X) \cap V_{\mathbb{Z}, f}^{0}\right)=\operatorname{Vol}\left(\mathcal{R}_{f}(X)\right)+ & O\left(\max \left\{\operatorname{Vol}\left(\overline{\mathcal{R}_{f}(X)}\right), 1\right\}\right) \\
=\frac{1}{\operatorname{det}\left(\Lambda_{f, w(f)}\right)} & \operatorname{Vol}\left(\Theta_{w(f)}\left(\mathcal{S}_{f}(X)\right)\right) \\
& +O_{f}\left(\max \left\{\operatorname{Vol}\left(\overline{\Theta_{w(f)}\left(\mathcal{S}_{f}(X)\right)}, 1\right\}\right),\right.
\end{aligned}
$$

where by Proposition 3.4, we know that

$$
\operatorname{det}\left(\Lambda_{f, w(f)}\right)= \begin{cases}s_{f} \alpha^{3} & \text { if } f \text { is irreducible } \\ s_{f} \beta^{3} / 8 & \text { if } f \text { is reducible. }\end{cases}
$$

Hence, it remains to compute the above volumes, which we shall do in Subsection 6.3. 
6.1. Proof of Proposition 6.1. Recall the notation from Proposition 3.2. By definition and Proposition 3.3, we then have a well-defined map

$$
\iota: V_{\mathbb{Z}, f}^{0} \longrightarrow \mathbb{Z}^{3} ; \quad \iota(F)=\left(L_{f}(F), L_{f, 1}(F), L_{f, 2}(F)\right) .
$$

Using Proposition 3.2, it is easy to verify that $\iota$ is in fact injective. We shall also need the following result due to Heath-Brown [18].

Lemma 6.3. Let $\xi\left(x_{1}, x_{2}, x_{3}\right)$ be a ternary quadratic form such that its corresponding matrix $M_{\xi}$ has non-zero determinant. For $B_{1}, B_{2}, B_{3}>0$, let $N_{\xi}\left(B_{1}, B_{2}, B_{3}\right)$ denote the number of tuples $\left(x_{1}, x_{2}, x_{3}\right) \in \mathbb{Z}^{3}$ such that

$$
\left|x_{1}\right| \leq B_{1},\left|x_{2}\right| \leq B_{2},\left|x_{3}\right| \leq B_{3}, \operatorname{gcd}\left(x_{1}, x_{2}, x_{3}\right)=1, \xi\left(x_{1}, x_{2}, x_{3}\right)=0 .
$$

Then, we have

$$
N_{\xi}\left(B_{1}, B_{2}, B_{3}\right) \ll_{\epsilon}\left(1+\left(B_{1} B_{2} B_{3} \cdot \frac{\operatorname{det}_{0}\left(M_{\xi}\right)^{2}}{\left|\operatorname{det}\left(M_{\xi}\right)\right|}\right)^{1 / 3+\epsilon}\right) d_{3}\left(\left|\operatorname{det}\left(M_{\xi}\right)\right|\right),
$$

where $\operatorname{det}_{0}\left(M_{\xi}\right)$ denotes the greatest common divisor of the $2 \times 2$ minors of $M_{\xi}$, and $d_{3}\left(\left|\operatorname{det}\left(M_{\xi}\right)\right|\right)$ is the number of ways to write $\left|\operatorname{det}\left(M_{\xi}\right)\right|$ as a product of three positive integers.

Proof. See [18, Corollary 2].

In what follows, consider $F \in \mathcal{S}_{f}(X) \cap V_{\mathbb{Z}, f}^{0}$, and for brevity, write

$$
\left(L, K, L_{1}, L_{2}\right)=\left(L_{f}(F), K_{f}(F), L_{f, 1}(F), L_{f, 2}(F)\right) .
$$

Since $\iota$ is injective, it is enough to estimate the number of choices for $\left(L, L_{1}, L_{2}\right)$. To that end, let us put $\mathcal{D}_{f}=\Delta(f)$. Recall from Propositions 3.2 and 3.3 that

$$
L, K, L_{1}, L_{2} \in \mathbb{Z} \text {, as well as } L_{1}^{2}-\mathcal{D}_{f} L_{2}^{2}=4 \alpha^{4}\left(L^{2}+4 K\right) / 9,
$$

which is non-zero by (1.7). By the definition of our height, we also have

$$
\begin{cases}L=O_{f}\left(X^{1 / 2}\right) \text { and } K=O_{f}(X) & \text { in all cases, } \\ L_{1}=O_{f}\left(X^{1 / 2}\right) \text { and } L_{2}=O_{f}\left(X^{1 / 2}\right) & \text { if } f \text { is irreducible. }\end{cases}
$$

The latter estimate holds by

$$
\begin{cases}(4.5),(4.2) & \text { if } f \text { is positive definite, } \\ (4.7),(4.8),(4.2), \text { and } 0 \leq t<t_{D_{f}} & \text { if } f \text { is indefinite and irreducible, }\end{cases}
$$

as well as the fact that $L_{1}$ and $L_{2}$ are linear in the coefficients of $F$. Finally, we shall write $d(-)$ for the divisor function.

Proof of Proposition 6.1: first claim. Suppose that $L^{2}+4 K=\square$. Then, we have

$$
L_{1}^{2}-\mathcal{D}_{f} L_{2}^{2}=U^{2} \text {, where } U \in \mathbb{N} \text { is such that } U=O_{f}\left(X^{1 / 2}\right) .
$$

If $f$ is reducible, then $\mathcal{D}_{f}=\square$ and so clearly there are

$$
O_{f}\left(\sum_{U=1}^{X^{1 / 2}} d\left(U^{2}\right)\right)=O_{f, \epsilon}\left(\sum_{U=1}^{X^{1 / 2}} X^{\epsilon}\right)=O_{f, \epsilon}\left(X^{1 / 2+\epsilon}\right)
$$

choices for the pair $\left(L_{1}, L_{2}\right)$. If $f$ is irreducible, then note that

$$
\left(L_{1} / n\right)^{2}-\mathcal{D}_{f}\left(L_{2} / n\right)^{2}=(U / n)^{2}, \text { where } n=\operatorname{gcd}\left(L_{1}, L_{2}, U\right),
$$


and applying Lemma 6.3 to the ternary quadratic form $\xi$ with matrix

$$
M_{\xi}=\left(\begin{array}{ccc}
1 & 0 & 0 \\
0 & -\mathcal{D}_{f} & 0 \\
0 & 0 & -1
\end{array}\right), \text { with }\left\{\begin{array}{l}
\operatorname{det}\left(M_{\xi}\right)=\mathcal{D}_{f} \\
\operatorname{det}_{0}\left(M_{\xi}\right)=1
\end{array}\right.
$$

we deduce from (6.3) that there are

$$
O_{f}\left(\sum_{n=1}^{X^{1 / 2}} N_{\xi}\left(\frac{X^{1 / 2}}{n}, \frac{X^{1 / 2}}{n}, \frac{X^{1 / 2}}{n}\right)\right)=O_{f, \epsilon}\left(\sum_{n=1}^{X^{1 / 2}}\left(1+\frac{X^{1 / 2+\epsilon}}{n^{1+\epsilon}}\right)\right)=O_{f, \epsilon}\left(X^{1 / 2+\epsilon}\right)
$$

choices for the pair $\left(L_{1}, L_{2}\right)$. In both cases, we see that there are

$$
O_{f}\left(X^{1 / 2}\right) \cdot O_{f, \epsilon}\left(X^{1 / 2+\epsilon}\right)=O_{f, \epsilon}\left(X^{1+\epsilon}\right)
$$

choices for $\left(L, L_{1}, L_{2}\right)$ in total, whence the claim.

Proof of Proposition 6.1: second claim. Suppose that $\left(L^{2}+4 K\right)\left(2 L^{2}-K\right) / \mathcal{D}_{f}=\square$. By Proposition 3.3, we may write

$$
\operatorname{gcd}\left(L^{2}+4 K, 4\left(2 L^{2}-K\right) / \mathcal{D}_{f}\right)=9 m a^{2}, \text { where } m, a \in \mathbb{N} \text { and } m \text { is square-free. }
$$

From the hypothesis, we then easily see that

$$
L^{2}+4 K=9 m U^{2} \text { and } 4\left(2 L^{2}-K\right) / \mathcal{D}_{f}=9 m V^{2}, \text { where } U, V \in \mathbb{N},
$$

as well as that $m$ divides $L$. In particular, a simple calculation yields

$$
L^{2}=m\left(U^{2}+\mathcal{D}_{f} V^{2}\right) \text {, whence } m W^{2}=U^{2}+\mathcal{D}_{f} V^{2} \text {, where } W \in \mathbb{Z} \text { with } L=m W \text {. }
$$

Now, suppose also that $L \neq 0$, in which case $m=O_{f}\left(X^{1 / 2}\right)$ by (6.3). Note also that

$$
m(W / n)^{2}=(U / n)^{2}+\mathcal{D}_{f}(V / n)^{2}, \text { where } n=\operatorname{gcd}(W, U, V) .
$$

Applying Lemma 6.3 to the ternary quadratic form $\xi_{m}$ with matrix

$$
M_{\xi_{m}}=\left(\begin{array}{ccc}
m & 0 & 0 \\
0 & -1 & 0 \\
0 & 0 & -\mathcal{D}_{f}
\end{array}\right), \text { with }\left\{\begin{array}{l}
\operatorname{det}\left(M_{\xi_{m}}\right)=m \mathcal{D}_{f} \\
\operatorname{det}_{0}\left(M_{\xi_{m}}\right)=\operatorname{gcd}\left(m, \mathcal{D}_{f}\right) \leq\left|\mathcal{D}_{f}\right|
\end{array}\right.
$$

we then see from (6.3) that there are

$$
\begin{aligned}
O_{f}\left(\sum_{n=1}^{X^{1 / 2} / m} N_{\xi_{m}}\left(\frac{X^{1 / 2}}{m n}, \frac{X^{1 / 2}}{m^{1 / 2} n}, \frac{X^{1 / 2}}{m^{1 / 2} n}\right)\right) & =O_{f, \epsilon}\left(\sum_{n=1}^{X^{1 / 2} / m}\left(1+\frac{X^{1 / 2+\epsilon}}{(m n)^{1+\epsilon}}\right) m^{\epsilon}\right) \\
& =O_{f, \epsilon}\left(\frac{X^{1 / 2}}{m^{1-\epsilon}}+\frac{X^{1 / 2+\epsilon}}{m}\right)
\end{aligned}
$$

choices for $(x, u, v)$ when $m$ is fixed. It follows that we have

$$
O_{f, \epsilon}\left(\sum_{m=1}^{X^{1 / 2}}\left(\frac{X^{1 / 2}}{m^{1-\epsilon}}+\frac{X^{1 / 2+\epsilon}}{m}\right)\right)=O_{f, \epsilon}\left(X^{1 / 2+\epsilon}\right)
$$

choices for $(m, x, u, v)$ and hence for $(L, K)$.

Next, regard $(L, K)$ as being fixed, and recall that

$$
L_{1}^{2}-\mathcal{D}_{f} L_{2}^{2}=T, \text { where } T=4 \alpha^{4}\left(L^{2}+4 K\right) / 9 .
$$


We claim that there are $O_{f}(d(T))$ choices for $\left(L_{1}, L_{2}\right)$. If $f$ is positive definite or if $f$ is reducible, then this is clear. If $f$ is indefinite and irreducible, then by Definition 5.2 as well as Propositions 3.1 and 4.3, we have

$$
F=\left(\Psi_{f} \circ \Phi^{(i)}\right)(L, K, t) \text {, where } 0 \leq t<t_{D_{f}} \text { and } i \in\{1,2,3,4\} .
$$

Since $\mathcal{D}_{f}>0$, we must have $L^{2}+4 K>0$ by the hypothesis, and so in fact $i \in\{1,2\}$. From the proof of Lemma 5.7, we know that

$$
L_{1}-\sqrt{D_{f}} L_{2}=(-1)^{i} \sqrt{T} e^{4 t} \text { and } L_{1}+\sqrt{D_{f}} L_{2}=(-1)^{i} \sqrt{T} e^{-4 t},
$$

which implies that

$$
L_{1}=(-1)^{i} \sqrt{T} \cosh (4 t) \text { and } L_{2}=(-1)^{i} \sqrt{T} \sinh (4 t) / \sqrt{D_{f}} .
$$

Since $t=O_{f}(1)$, we then deduce that indeed there are $O_{f}(d(T))$ choices for $\left(L_{1}, L_{2}\right)$. Using the bound $d(T)=O_{\epsilon}\left(T^{\epsilon}\right)=O_{f, \epsilon}\left(X^{\epsilon}\right)$, we conclude that there are

$$
O_{f, \epsilon}\left(X^{1 / 2+\epsilon}\right) \cdot O_{f, \epsilon}\left(X^{\epsilon}\right)=O_{f, \epsilon}\left(X^{1 / 2+\epsilon}\right)
$$

choices for $\left(L, L_{1}, L_{2}\right)$ in total, whence the claim.

Proof of Proposition 6.1: third claim. Suppose that $L=0$ and that $F$ is in the shape as in (3.1). Using Proposition 3.2, we then deduce that

$$
C=(-12 \gamma A+3 \beta B) /(2 \alpha) \text {, and so } K=-9 \mathcal{D}_{f}\left(\alpha B^{2}-4 \beta A B+16 \gamma A^{2}\right) /\left(4 \alpha^{3}\right) .
$$

Hence

$$
\left(L^{2}+4 K\right)\left(2 L^{2}-K\right) / 81 \mathcal{D}_{f}=-4 \mathcal{D}_{f}\left(\alpha B^{2}-4 \beta A B+16 \gamma A^{2}\right)^{2} /\left(4 \alpha^{3}\right)^{2},
$$

from which it follows that the above expression is a square if and only if $-\mathcal{D}_{f}$ is a square. This also follows immediately from the observation that the above product is equal to $-4 K^{2} / \mathcal{D}_{f}$ in this case.

We now suppose that $-\Delta(f)=\square$, so in particular $f$ is positive definite. $F$ is then determined by $(A, B) \in \mathbb{Z}^{2}$, and that $|K| \leq X$ implies

$$
\left|\left(B-\frac{2 \beta}{\alpha} A\right)^{2}-\frac{4 \mathcal{D}_{f}}{\alpha^{2}} A^{2}\right| \ll_{f} X .
$$

Hence there are $O_{f}(X)$ choices for $(A, B)$. It follows that the claim holds.

6.2. Proof of Proposition 6.2. By Lemma 3.8 and Proposition 6.1, we have

$$
\#\left\{F \in \mathcal{S}_{f}(X) \cap V_{\mathbb{Z}, f}^{0}: F \text { is reducible of type } 1\right\}=O_{f, \epsilon}\left(X^{1+\epsilon}\right),
$$

whence it is enough to consider the reducible forms in $\mathcal{S}_{f}(X) \cap V_{\mathbb{Z}, f}^{0}$ of type 2 ; recall Definition 3.7. By definition, such a form has the shape

$$
F(x, y)=p_{2} q_{2} x^{4}+\left(p_{2} q_{1}+p_{1} q_{2}\right) x^{3} y+\left(p_{2} q_{0}+p_{1} q_{1}+p_{0} q_{2}\right) x^{2} y^{2}+(*) x y^{3}+(*) y^{4},
$$

where $p_{2}, p_{1}, p_{0}, q_{2}, q_{1}, q_{0} \in \mathbb{Z}$, and we have

$$
p_{0}=\left(\beta p_{1}-2 \gamma p_{2}\right) /(2 \alpha) \text { and } q_{0}=\left(\beta q_{1}-2 \gamma q_{2}\right) /(2 \alpha)
$$

by Lemma 3.10. We have the condition

$$
\begin{aligned}
&\left|\left(\alpha p_{1}^{2}-2 \beta p_{1} p_{2}+4 \gamma p_{2}^{2}\right) / \alpha\right|,\left|\left(\alpha q_{1}^{2}-2 \beta q_{1} q_{2}+4 \gamma q_{2}^{2}\right) / \alpha\right| \\
&\left|p_{2}\right|,\left|\alpha p_{1}-\beta p_{2}\right|,\left|q_{2}\right|,\left|\alpha q_{1}-\beta q_{2}\right| \geq 1
\end{aligned}
$$


since the above numbers are all integers. Using Proposition 3.2 (a), we compute that

$$
\frac{L_{f}(F)^{2}+4 K_{f}(F)}{9}=\frac{\alpha p_{1}^{2}-2 \beta p_{1} p_{2}+4 \gamma p_{2}^{2}}{\alpha} \cdot \frac{\alpha q_{1}^{2}-2 \beta q_{1} q_{2}+4 \gamma q_{2}^{2}}{\alpha} .
$$

Now, by the definition of our height, we clearly have

$$
\left|\left(\alpha p_{1}^{2}-2 \beta p_{1} p_{2}+4 \gamma p_{2}^{2}\right) / \alpha\right|,\left|\left(\alpha q_{1}^{2}-2 \beta q_{1} q_{2}+4 \gamma q_{2}^{2}\right) / \alpha\right| \leq X .
$$

Observe also that

$$
p_{2} q_{2}, p_{2} q_{1}+p_{1} q_{2}, p_{1} q_{1}=O_{f}\left(X^{1 / 2}\right) \text { if } f \text { is indefinite and irreducible }
$$

by (4.7), (4.8), (4.2), and the bound $0 \leq t<t_{D_{f}}$. We then deduce that

$$
\#\left\{F \in \mathcal{S}_{f}(X) \cap V_{\mathbb{Z}, f}^{0}: F \text { is reducible of type } 2\right\} \leq \#\left(\mathcal{R}_{f}^{\prime}(X) \cap \mathbb{Z}^{4}\right),
$$

where we define

$$
\mathcal{R}_{f}^{\prime}(X)=\left\{\left(p_{2}, p_{1}, q_{2}, q_{1}\right) \in \mathbb{R}^{4}:(6.5),(6.6), \text { and }(6.7)\right\} .
$$

It is clear that this set is bounded and semi-algebraic. Hence, we may apply Proposition 5.1 to estimate the number of integral points it contains.

6.2.1. The case when $f$ is irreducible. Let us define

$$
\mathcal{R}_{f}^{\prime \prime}(X)=\mathcal{L}_{D_{f}}\left(\mathcal{R}_{f}^{\prime}(X)\right), \text { where } \mathcal{L}_{D_{f}}=\left(\begin{array}{cccc}
\sqrt{D_{f}} & 0 & 0 & 0 \\
-\beta & \alpha & 0 & 0 \\
0 & 0 & \sqrt{D_{f}} & 0 \\
0 & 0 & -\beta & \alpha
\end{array}\right) .
$$

Applying Proposition 5.1, we then obtain

$$
\begin{aligned}
\#\left(\mathcal{R}_{f}^{\prime}(X) \cap \mathbb{Z}^{4}\right) & =\operatorname{Vol}\left(\mathcal{R}_{f}^{\prime}(X)\right)+O\left(\max \left\{\operatorname{Vol}\left(\overline{\mathcal{R}_{f}(X)}, 1\right\}\right)\right. \\
& =\frac{1}{\operatorname{det}\left(\mathcal{L}_{D_{f}}\right)} \operatorname{Vol}\left(\mathcal{R}_{f}^{\prime \prime}(X)\right)+O_{f}\left(\max \left\{\operatorname{Vol}\left(\overline{\mathcal{R}_{f}^{\prime \prime}(X)}\right), 1\right\}\right)
\end{aligned}
$$

For any $\left(u_{2}, u_{1}, v_{2}, v_{1}\right) \in \mathcal{R}_{f}^{\prime \prime}(X)$, from (6.5) and (6.6), we deduce that

$$
\left|u_{2}\right|,\left|u_{1}\right|,\left|v_{2}\right|,\left|v_{1}\right| \geq 1
$$

as well as that

$$
\begin{cases}1 \leq\left|u_{1}^{2}+u_{2}^{2}\right|,\left|v_{1}^{2}+v_{2}^{2}\right| \leq \alpha^{4} X & \text { if } f \text { is positive definite } \\ 1 \leq\left|u_{1}^{2}-u_{2}^{2}\right|,\left|v_{1}^{2}-v_{2}^{2}\right| \leq \alpha^{4} X & \text { if } f \text { is indefinite. }\end{cases}
$$

This, together with (6.7), implies that in fact

$$
1 \leq\left|u_{2}\right|,\left|u_{1}\right|,\left|v_{2}\right|,\left|v_{1}\right|,\left|u_{2} v_{2}\right|,\left|u_{1} v_{1}\right| \ll_{f} X^{1 / 2} .
$$

We then compute that

$$
\begin{aligned}
& \operatorname{Vol}\left(\mathcal{R}_{f}^{\prime \prime}(X)\right)=O_{f}\left(\prod_{i=1}^{2} \int_{1}^{X^{1 / 2} / v_{i}} d u_{i} d v_{i}\right)=O_{f}\left(X(\log X)^{2}\right), \\
& \operatorname{Vol}\left(\overline{\mathcal{R}_{f}^{\prime \prime}(X)}\right)=O_{f}(X \log X) .
\end{aligned}
$$

The claim now follows from (6.4) and (6.8). 
6.2.2. The case when $f$ is reducible. Let us define

$$
\mathcal{R}_{f}^{\prime \prime}(X)=\mathcal{L}_{0, D_{f}}\left(\mathcal{R}_{f}^{\prime}(X)\right) \text {, where } \mathcal{L}_{0, D_{f}}=\left(\begin{array}{cccc}
1 & 1 & 0 & 0 \\
-1 & 1 & 0 & 0 \\
0 & 0 & 1 & 1 \\
0 & 0 & -1 & 1
\end{array}\right)\left(\begin{array}{cccc}
\sqrt{D_{f}} & 0 & 0 & 0 \\
-\beta & \alpha & 0 & 0 \\
0 & 0 & \sqrt{D_{f}} & 0 \\
0 & 0 & -\beta & \alpha
\end{array}\right) .
$$

Since $D_{f}=\square$ in this case, we see that

$$
\mathcal{L}_{0, D_{f}}\left(\mathcal{R}_{f}^{\prime}(X) \cap \mathbb{Z}^{4}\right) \subset \mathcal{R}_{f}^{\prime \prime}(X) \cap \mathbb{Z}^{4} \text { and so } \#\left(\mathcal{R}_{f}^{\prime}(X) \cap \mathbb{Z}^{4}\right) \leq \#\left(\mathcal{R}_{f}^{\prime \prime}(X) \cap \mathbb{Z}^{4}\right) .
$$

Now, applying Proposition 5.1, we have

$$
\#\left(\mathcal{R}_{f}^{\prime \prime}(X) \cap \mathbb{Z}^{4}\right)=\operatorname{Vol}\left(\mathcal{R}_{f}^{\prime \prime}(X)\right)+O\left(\max \left\{\operatorname{Vol}\left(\overline{\mathcal{R}_{f}^{\prime \prime}(X)}\right), 1\right\}\right) .
$$

For any $\left(z_{1}, z_{2}, z_{3}, z_{4}\right) \in \mathcal{R}_{f}^{\prime \prime}(X)$, the conditions (6.5) and (6.6) imply that

$$
\left|z_{1}\right|,\left|z_{2}\right|,\left|z_{3}\right|,\left|z_{4}\right| \geq 1 \text { and }\left|z_{1} z_{2} z_{3} z_{4}\right| \leq \alpha^{4} X
$$

which is analogous to (6.9). We then compute that

$$
\begin{aligned}
& \operatorname{Vol}\left(\mathcal{R}_{f}^{\prime \prime}(X)\right)=O_{f}\left(\int_{1}^{X} \int_{1}^{\frac{X}{z_{4}}} \int_{1}^{\frac{X}{z_{3} z_{4}}} \int_{1}^{\frac{X}{z_{2} z_{3} z_{4}}} d z_{1} d z_{2} d z_{3} d z_{4}\right)=O_{f}\left(X(\log X)^{3}\right), \\
& \operatorname{Vol}\left(\overline{\mathcal{R}_{f}^{\prime \prime}(X)}\right)=O_{f}\left(X(\log X)^{2}\right) .
\end{aligned}
$$

The claim now follows from (6.4) and (6.8).

6.3. Proof of Theorem 1.2. We have already proven part (d). To prove parts (a) through (c), it remains to compute the volumes in (6.2).

6.3.1. The case when $f$ is positive definite. We have

$$
\operatorname{Vol}\left(\Theta_{1}\left(\mathcal{S}_{f}(X)\right)\right)=\frac{8 \alpha^{3}}{D_{f}^{3 / 2}} \cdot \frac{1}{18} \cdot \operatorname{Vol}\left(\Omega^{+}(X) \times[-\pi / 4, \pi / 4)\right)
$$

by Lemma 4.1 and Proposition 4.2 (b), as well as

$$
\operatorname{Vol}\left(\Omega^{+}(X) \times[-\pi / 4, \pi / 4)\right)=\int_{-X^{1 / 2}}^{X^{1 / 2}} \int_{-L^{2} / 4}^{X} \frac{\pi}{2} d K d L=\frac{13 \pi}{12} X^{3 / 2} .
$$

Observe also that

$$
\operatorname{Vol}\left(\overline{\Theta_{1}\left(\mathcal{S}_{f}(X)\right)}\right)=O_{f}(X)
$$

because $\Theta_{1}\left(\mathcal{S}_{f}(X)\right)$ lies in the cube centered at the origin of side length $O_{f}\left(X^{1 / 2}\right)$ by (4.5) and (4.2). We then deduce part (a) from (6.1) and (6.2).

6.3.2. The case when $f$ is reducible. We have

$$
\operatorname{Vol}\left(\Theta_{2}\left(\mathcal{S}_{f}(X)\right)\right)=\frac{1}{18} \cdot 2 \cdot \operatorname{Vol}\left(\Omega^{0}(X) \times\left[t_{f, 1}, t_{f, 2}\right]\right)
$$

by Proposition 4.4, as well as

$$
\operatorname{Vol}\left(\Omega^{0}(X) \times\left[t_{f, 1}, t_{f, 2}\right]\right)=\int_{-X^{1 / 2}}^{X^{1 / 2}} \int_{-X}^{X} \frac{1}{4} \log \left(\frac{20 X}{9}\right) d K d L=X^{3 / 2} \log (20 X / 9) .
$$

We then deduce part (b) from Lemma 6.4 below as well as (6.1) and (6.2).

Lemma 6.4. We have $\operatorname{Vol}\left(\overline{\Theta_{2}\left(\mathcal{S}_{f}(X)\right)}\right)=O_{f}\left(X^{3 / 2}\right)$. 
Proof. By Definition 5.2, an element in $\Theta_{2}\left(\mathcal{S}_{f}(X)\right)$ takes the form

$$
(A, B, C)=\left(\Theta_{2} \circ \Phi_{f}\right)(L, K, t), \text { where }(L, K, t) \in \Omega^{0}(X) \times\left[t_{f, 1}, t_{f, 2}\right] .
$$

Let us recall that

$$
|L| \leq X^{1 / 2},|K| \leq X, 4 t_{f, 1}=-\log 8,4 t_{f, 2}=\log (5 X / 18) .
$$

Then, from (4.11), we see that 1-dimensional projections of $\Theta_{2}\left(\mathcal{S}_{f}(X)\right)$ have lengths of order $O_{f}(X)$. As for the 2-dimensional projections, note that (5.1) and (6.10) yield

$$
|C|=\beta^{2} e^{4 t} \text { and } 1 \ll_{f}|C| \ll_{f} X,
$$

as well as the estimates

$$
\left|B-\frac{6 \alpha^{2} C}{\beta^{2}}\right| \leq \frac{1}{2} X^{1 / 2} \text { and }\left|A-\frac{\alpha^{4} C}{\beta^{4}}\right| \leq \frac{5}{144|C|} X+\frac{\alpha^{2}}{2 \beta^{2}} X^{1 / 2} .
$$

Hence, the projections of $\Theta_{2}\left(\mathcal{S}_{f}(X)\right)$ onto the $B C$-plane and $A C$-plane, respectively, have areas bounded by

$$
O_{f}\left(\int_{1}^{X} X^{1 / 2} d C\right) \text { and } O_{f}\left(\int_{1}^{X}\left(\frac{1}{C} X+X^{1 / 2}\right) d C\right) .
$$

Similarly, from (5.1) and (6.10), we deduce that

$$
|2 B-L|=12 \alpha^{2} e^{4 t}, 1 \ll_{f}|2 B-L| \ll_{f} X,|B| \ll_{f} X,
$$

as well as the estimate

$$
\left|A-\frac{\alpha^{2} B}{6 \beta^{2}}\right| \leq \frac{5 \alpha^{2}}{12 \beta^{2}}\left(\frac{1}{|2 B-L|} X+X^{1 / 2}\right) .
$$

Note that $|L| \leq X^{1 / 2}$ also implies that

$$
|2 B-L| \geq|2| B|-| L|| \geq 2|B|-X^{1 / 2} \text { when }|B| \geq X^{1 / 2} / 2 .
$$

Hence, the projection of $\Theta_{2}\left(\mathcal{S}_{f}(X)\right)$ onto the $A B$-plane has area bounded by

$$
O_{f}\left(\int_{0}^{1+X^{1 / 2} / 2}\left(X+X^{1 / 2}\right) d B+\int_{1+X^{1 / 2} / 2}^{X}\left(\frac{1}{2 B-X^{1 / 2}} X+X^{1 / 2}\right) d B\right) .
$$

It follows that all of the 2-dimensional projections of $\Theta_{2}\left(\mathcal{S}_{f}(X)\right)$ have areas of order $O_{f}\left(X^{3 / 2}\right)$, and this proves the lemma.

6.3.3. The case when $f$ is indefinite and irreducible. We have

$$
\operatorname{Vol}\left(\Theta_{1}\left(\mathcal{S}_{f}(X)\right)\right)=\frac{8 \alpha^{3}}{D_{f}^{3 / 2}} \cdot \frac{1}{18} \cdot 2 \cdot\left(\operatorname{Vol}\left(\Omega^{+}(X) \times\left[0, t_{D_{f}}\right)\right)+\operatorname{Vol}\left(\Omega^{-}(X) \times\left[0, t_{D_{f}}\right)\right)\right)
$$

by Lemma 4.1 and Proposition 4.3, as well as

$$
\begin{aligned}
& \operatorname{Vol}\left(\Omega^{+}(X) \times\left[0, t_{D_{f}}\right)\right)=\int_{-X^{1 / 2}}^{X^{1 / 2}} \int_{-L^{2} / 4}^{X} t_{D_{f}} d K d L=\frac{13 t_{D_{f}}}{6} X^{3 / 2}, \\
& \operatorname{Vol}\left(\Omega^{-}(X) \times\left[0, t_{D_{f}}\right)\right)=\int_{-X^{1 / 2}}^{X^{1 / 2}} \int_{-X}^{-L^{2} / 4} t_{D_{f}} d K d L=\frac{11 t_{D_{f}}}{6} X^{3 / 2},
\end{aligned}
$$

Observe also that

$$
\operatorname{Vol}\left(\overline{\Theta_{1}\left(\mathcal{S}_{f}(X)\right)}\right)=O_{f}(X)
$$


because $\Theta_{1}\left(\mathcal{S}_{f}(X)\right)$ lies in the cube centered at the origin of side length $O_{f}\left(X^{1 / 2}\right)$ by (4.7), (4.8), (4.2), and the bound on $t$. We then deduce part (c) from (6.1) and (6.2).

\section{ACKnowledgments}

The first-named author was partially supported by the China Postdoctoral Science Foundation Special Financial Grant (grant number: 2017T100060). We would like to thank the referee for many useful suggestions which helped improve the exposition of the paper significantly.

\section{REFERENCES}

[1] A. Altug, A. Shankar, I. Varma, and K. Wilson, The number of quartic $D_{4}$-fields ordered by conductor, arXiv:1704.01729v1 [math.NT].

[2] M. Bhargava, Higher composition laws III. The parametrization of quartic rings, Ann. of Math. 159 (2004), no. 3, 1329-1360.

[3] M. Bhargava, Higher composition laws IV: The parametrization of quintic rings, Ann. of Math. 167 (2008), no.1, 53-94.

[4] M. Bhargava and A. Shankar, Binary quartic forms having bounded invariants, and the boundedness of the average rank of elliptic curves, Ann. of Math. 181 (2015), no. 1, 191-242.

[5] M. Bhargava, A. Shankar, and J. Tsimerman, On the Davenport-Heilbronn theorems and second order terms, Invent. Math. 193 (2013), no. 2, 193-439 .

[6] M . Bhargava and A. Shnidman, On the number of cubic orders of bounded discriminant having automorphism group $C_{3}$, and related problems, Algebra and Number Theory 8 (2014), no. 1, 53-88.

[7] B. J. Birch and J. R. Merriman, Finiteness theorems for binary forms with given discriminant, Proc. London Math. Soc. 24 (1972), no. 3, 385-394.

[8] A. Brumer, The average rank of elliptic curves. I, Invent. Math. 109 (1992), no. 1, 445-472.

[9] K. Conrad, Galois groups of cubics and quartics (not in characteristic 2), Online notes, retrieved 24 Nov, 2017. http://www.math.uconn.edu/ kconrad/blurbs/galoistheory/cubicquartic.pdf

[10] J. Cremona, Reduction of binary cubic and quartic forms, LMS J. Comput. Math. 2 (1999), 64-94.

[11] H. Davenport, On a principle of Lipschitz, J. London Math. Soc. 26 (1951), no. 3, 179-183.

[12] H. Davenport, On the class-number of binary cubic forms I, J. London Math. Soc. 26 (1951), no. $3,183-192$.

[13] H. Davenport, On the class-number of binary cubic forms II. J. London Math. Soc. 26 (1951), no. 3, 192-198.

[14] H. Davenport and H. Heilbronn, On the density of discriminants of cubic fields. II, Proc. Roy. Soc. London Ser. A 322 (1971), no. 1551, 405-420.

[15] B. N. Delone and D. K. Faddev, The theory of irrationalities of the third degree. Translations of Mathematical Monographs, Vol. 10 American Mathematical Society, Providence, R.I. 1964.

[16] C. F. Gauss, Disquisitiones Arithmeticae, 1801.

[17] D. R. Heath-Brown, The average analytic rank of elliptic curves, Duke Math. J. 122 (2004), no. 3, 591-623.

[18] D. R. Heath-Brown, The density of rational points on curves and surfaces, Ann. of Math. 155 (2002), no. 2, 553-598.

[19] Z. Klagsbrun and R. Lemke-Oliver, The distribution of the Tamagawa ratio in the family of elliptic curves with a two-torsion point, Res. Math. Sci. 1 (2014), no. 1, 1-10.

[20] J. Nakagawa, Binary forms and orders of algebraic number fields, Invent. Math. 97 (1989), no. 2, 219-235. Erratum: ibid. 105 (1991), no. 2, 443.

[21] M. Newman, Integral matrices, Pure and Appl. Math. (S. Eilenberg and P. A. Smith, eds.), vol. 45, Academic Press, New York, 1972.

[22] C. L. Siegel, The average measure of quadratic forms with given determinant and signature, Ann. of Math. 45 (1944), no. 4, 667-685. 
[23] T. Taniguchi and F. Thorne, Secondary terms in counting functions for cubic fields, Duke Math. J. 162 (2013), no. 13, 2451-2508.

[24] C. Tsang and S. Y. Xiao, The number of quartic $D_{4}$-fields with monogenic cubic resolvent ordered by conductor, arXiv:1712.08552v3 [math.NT].

[25] M. Wood, Quartic rings associated to binary quartic forms, Int. Math. Res. Not. IMRN 2012, no. $6,1300-1320$.

[26] M. P. Young, Low-lying zeros of families of elliptic curves, J. Amer. Math. Soc. 19 (2006), no. 1, 205-250.

[27] S. Y. Xiao, On binary cubic and quartic forms, arXiv:1610.09208v2 [math.NT].

[28] S. Y. Xiao, On binary quartic forms with vanishing J-invariant, arXiv:1712.09091v2 [math.NT].

Yau Mathematical Sciences Center, Tsinghua University, Beijing, P. R. China

E-mail address: sinyitsang@math.tsinghua.edu.cn

Mathematical Institute, University of Oxford, Andrew Wiles Building, Radcliffe Observatory Quarter, Woodstock Road, Oxford, OX2 6GG

E-mail address: stanley.xiao@maths.ox.ac.uk 